1

2

4

5

\title{
Lipid hydroperoxides promote muscle atrophy through lysosomal amplification
}

Hiroaki Eshima, ${ }^{1-3}$ Piyarat Siripoksup,,${ }^{1,4}$ Justin L. Shahtout, ${ }^{1,4}$ MacKenzie J. Pearson, ${ }^{5}$ Ziad

S. Mahmassani, ${ }^{1,2,4}$ Patrick J. Ferrara,,${ }^{1,2,6}$ Alexis W. Lyons, ${ }^{1}$ J. Alan Maschek, ${ }^{1,6,7}$ Alek D.

Peterlin, ${ }^{1,6}$ Anthony R. P. Verkerke, ${ }^{1,6}$ Jordan M. Johnson, ${ }^{1,6}$ Anahy Salcedo, ${ }^{1}$ Jonathan J.

Petrocelli, ${ }^{1,4}$ Ethan J. Anderson, ${ }^{8}$ Sihem Boudina, ${ }^{1,2,6}$ Qitao Ran, ${ }^{9}$ James E. Cox, ${ }^{1,7,10}$ Micah

J. Drummond, ${ }^{1,2,4}$ Katsuhiko Funai, ${ }^{1,2,4,6 *}$

${ }^{1}$ Diabetes \& Metabolism Research Center, University of Utah, Salt Lake City, Utah, USA ${ }^{2}$ Molecular Medicine Program, University of Utah, Salt Lake City, Utah, USA

${ }^{3}$ Department of International Tourism, Nagasaki International University, Sasebo, Nagasaki, Japan

${ }^{4}$ Department of Physical Therapy \& Athletic Training, University of Utah, Salt Lake City, Utah, USA

${ }^{5}$ Sciex, Framingham, MA, USA

${ }^{6}$ Department of Nutrition \& Integrative Physiology, University of Utah, Salt Lake City, Utah, USA

${ }^{7}$ Metabolomics Core Research Facility, University of Utah, Salt Lake City, Utah, USA ${ }^{8}$ Fraternal Order of Eagles Diabetes Research Center, University of lowa, lowa City, lowa, USA

${ }^{9}$ Department of Cell Systems and Anatomy, University of Texas Health Science Center at San Antonio, San Antonio, Texas, USA

${ }^{10}$ Department of Biochemistry, University of Utah, Salt Lake City, Utah, USA

${ }^{*}$ Corresponding Author:

Katsuhiko Funai, Ph.D.

Diabetes \& Metabolism Research Center 
bioRxiv preprint doi: https://doi.org/10.1101/2021.12.17.473200; this version posted December 20, 2021. The copyright holder for this

preprint (which was not certified by peer review) is the author/funder, who has granted bioRxiv a license to display the preprint in perpetuity. It is made available under aCC-BY-NC-ND 4.0 International license.

15 N 2030 E, Salt Lake City, UT 84112

Phone: (801) 585-1781

30

Fax: (801) 585-0701

31

Email: kfunai@health.utah.edu 


\section{Abstract}

33 Reactive oxygen species (ROS) is a cardinal feature of skeletal muscle atrophy. However,

34 ROS refers to a collection of radical molecules whose cellular signals are vast, and it is unclear which downstream consequences of ROS are responsible for the loss of muscle mass and strength. ${ }^{1,2}$ Here we show that lipid hydroperoxides $(\mathrm{LOOH})$ are increased with age and disuse, and the accumulation of $\mathrm{LOOH}$ by suppression of glutathione peroxidase 4 (GPx4) is sufficient to augment muscle atrophy. Strikingly, genetic and pharmacologic suppression of muscle $\mathrm{LOOH}$ robustly prevented the reduction of both muscle mass and force-generating capacity. $\mathrm{LOOH}$ promoted atrophy in a lysosomal-dependent, proteasomal-independent manner, and the suppression of autophagic machinery was sufficient to prevent muscle atrophy and weakness. Indeed, the lysosome is essential for the amplification of $\mathrm{LOOH}$ induced by oxidative stress. Our findings provide novel insights for the role of $\mathrm{LOOH}$ in muscle atrophy including a therapeutic implication by pharmacologic suppression.

\section{Main}

Loss of muscle mass and function with age is detrimental to health and quality of life.$^{3,4} \mathrm{~A}$ pharmacologic therapy for muscle loss does not exist, and current diet or exercise therapeutic approaches are often ineffective or unfeasible. Oxidative stress has been implicated in muscle atrophy by accelerating proteolysis, ${ }^{2}$ but the exact mechanism by which ROS contributes to the decrease in muscle mass and strength is not well understood. Lipid hydroperoxide ( $\mathrm{LOOH}$ ) is a class of lipid ROS molecules that has been implicated in cell damage, particularly as a trigger to induce ferroptosis, a non-apoptotic form of regulated cell death. ${ }^{5,6} \mathrm{LOOH}$ propagation is endogenously suppressed by GPx4 that catalyzes the reaction by which $\mathrm{LOOH}$ is reduced to its nonreactive hydroxyl metabolite. ${ }^{7}$ However, the role of $\mathrm{LOOH}$ in skeletal muscle is not well understood..$^{8,9}$ 
We first evaluated the changes in skeletal muscle $\mathrm{LOOH}$ with aging. In humans and in mice, aging promoted a reduction in the expression of GPx4 in skeletal muscle (Fig. 1a\&b). To examine the changes in skeletal muscle $\mathrm{LOOH}$ landscape with age, we performed a comprehensive oxidolipidomic analysis in muscle samples from young (4 months) and old (20 months) mice (Fig. 1c\&d). We detected over 300 species of oxidized lipids with an effect distribution that was highly class-dependent. Among these, age had the most robust effect on oxidized phosphatidylethanolamine (Fig. 1C, red), a class of lipids that have been implicated as a potential lipid signal to induce ferroptosis. ${ }^{10}$ Among the top ten oxidized lipid species whose abundance was most robustly increased with age, six of them were oxidized phosphatidylethanolamine (Figure 1d), and they were substantially more highly abundant compared to other oxidized lipids. $\mathrm{LOOH}$ can be indirectly assessed by quantifying LOOH adducts such as 4-hydroxynonenal (4-HNE) and malondialdehyde c).

Disuse also promotes skeletal muscle atrophy that is likely contributed by ROS. ${ }^{2}$ To model disuse atrophy, mice underwent a hindlimb unloading $(\mathrm{HU})$ procedure as previously described. ${ }^{11,12}$ As expected, HU induced muscle atrophy and weakness (Fig. 1e\&f) concomitant to reduction in body and lean mass (Extended Data Fig. 1d-f). Disuse robustly elevated muscle LOOH levels (Fig. 1g, Extended Data Fig. 1g) without significant changes in mitochondrial bioenergetics (Extended Data Fig. 1h-j). An increase in muscle LOOH preceded atrophy, consistent with the notion that $\mathrm{LOOH}$ may trigger mechanisms to promote loss of muscle mass.

Next, we tested our hypothesis that $\mathrm{LOOH}$ contributes to muscle atrophy using and markers of ferroptosis (Extended Data Fig. 2a-f) concomitant with a decrease in myotube diameter (Fig. 1i\&j). We also recapitulated these findings with erastin (system $\mathrm{X}_{c}^{-}$ inhibitor that suppresses glutathione synthesis) (Fig. 1i\&j and Extended Data Fig. 2g-k) and 
RSL3 (GPx4 inhibitor) (Fig. 1i\&j and Extended Data Fig. 2l-o), commonly used acute pharmacological interventions to elevate intracellular $\mathrm{LOOH}$. We then translated these findings in vivo with global heterozygous GPx4 knockout mice $\left(\mathrm{GP} \times 4^{+/-}\right)$. Germline deletion of GPx4 is embryonically lethal, ${ }^{13}$ but $\mathrm{GP} \times 4^{+/-}$mice appear normal and do not have an observable muscle phenotype at baseline. ${ }^{7,14}$ We studied 4 months (young) and 20 months (old) $\mathrm{GPX}^{+/-}$and wildtype littermates with or without HU (Extended Data Fig. 3a-d). In young mice, GPx4 haploinsufficiency augmented the loss in muscle mass induced by HU (Fig. 2a and Extended Data Fig. 3e). However, muscle masses between old GPx4 ${ }^{+/-}$and wildtype mice were not different. We interpret these findings to mean that disuse in old mice promotes an increase in $\mathrm{LOOH}$ that has already reached a maximally effective threshold with age such that GPx4 deletion had no further effect. In support of this, we saw no differences in 4-HNE or MDA levels between old GPx4 ${ }^{+/}$and wildtype mice (Fig. $2 \mathrm{~b}$ and Extended Data Fig. 3f\&g). GPx4 haploinsufficiency did not alter force-generating capacity (Fig. 2c and Extended Data Fig. 3h-j).

Because GPx4 is expressed globally, we also studied mice with skeletal musclespecific tamoxifen-inducible GPx4 knockout (GPx4-MKO) (Fig. 2d and Extended Fig. 4a). ${ }^{15}$ Consistent with GPx4+/- mice, GPx4-MKO mice were also more prone to developing disuse-induced skeletal muscle atrophy (Fig. 2e\&f and Extended Data Fig. 4b-f) concomitant to elevated LOOH (Fig. $2 g$ and Extended Data Fig. 4g\&h), suggesting that loss of GPx4 in muscle augments atrophy in a cell-autonomous manner. Histological analyses revealed that reduced muscle mass was consistent with reduced cross-sectional area of myofibers regardless of fiber-type compositions (Fig. 2h\&i and Extended Data Fig. 4i\&j). These data implicate that $\mathrm{LOOH}$ directly reduces muscle cell size in vivo.

GPx4 primarily neutralizes LOOH but it also exhibits some activity towards other peroxides. ${ }^{16}$ To confirm that the effects of GPx4 deletion to promote atrophy is specific to $\mathrm{LOOH}$, we diminished the ability of cells to incorporate polyunsaturated fatty acids (PUFAs) into phospholipids by deleting lysophosphatidylcholine acyltransferase 3 (LPCAT3). ${ }^{17-19}$ 
112 LPCAT3 is an enzyme of Lands cycle that preferentially acylates lysophospholipids with

113 PUFAs, and thus an essential component of ferroptosis ${ }^{10}$. Indeed, LPCAT3 KD rescued the

114 increase in 4-HNE induced by GPx4 KD (Fig. 2j and Extended Data Fig. 5a-c).

115 Remarkably, deletion of LPCAT3 KD completely restored the reduction in myotube

116 diameter induced by GPx4 KD (Fig. 2k\&l). Similarly, LPCAT3 deletion also prevented

$117 \mathrm{LOOH}$ and cell death induced by erastin (Extended Data Fig. 5d-h). These findings indicate

118 that muscle atrophy induced by loss of GPx4 or erastin treatment is due to the

119 accumulation of $\mathrm{LOOH}$ and not other peroxides.

120 Would suppression of $\mathrm{LOOH}$ be sufficient to ameliorate skeletal muscle atrophy? We

121 studied young (4 months) and old (20 months) global GPx4-overexpressing (GPx4Tg)

122 mice ${ }^{20}$ with or without HU (Extended Data Fig. 6a-e). Strikingly, both young and old

123 GPx4Tg mice were resistant to disuse-induced muscle atrophy (Fig. 3a and Extended Data

124 Fig. $6 \mathrm{f} \& \mathrm{~g})$. Perhaps even more impactful was the effect of GPx4 overexpression on skeletal

125 muscle force-generating capacity such that, in both young and old, GPx4 overexpression

126 robustly protected mice from muscle weakness induced by HU (Fig. 3b and Extended Data

127 Fig. 6h-j). These findings are in contrast to our experiments in $\mathrm{GPx}^{+/-}$mice where muscle

128 mass phenotype was only present in the young mice (Fig. 2a) and no phenotype on muscle

129 strength (Fig. 2b). Consistent with the notion that GPx4 overexpression acts on LOOH, HU-

130 induced increase in 4-HNE was completely suppressed in GPx4Tg mice (Fig. 3c and

131 Extended Data Fig. 6k). We also found that the protection from muscle atrophy was

132 explained by greater myofiber cross-sectional area regardless of fiber-type (Fig. 3d\&e and

133 Extended Data Fig. 6l\&m).

134 Next, we explored opportunities to pharmacologically suppress LOOH to prevent

135 muscle atrophy. Ferrostatin-1 is an inhibitor of ferroptosis that is widely used to study

$136 \mathrm{LOOH} .^{21,22}$ Indeed, incubation of cells with ferrostatin-1 was sufficient to suppress LOOH

137 induced by GPx4 KD (Fig. 3f and Extended Data Fig. 7a) concomitant with protection from

138 myotube atrophy (Fig. 3g\&h and Extended Data Fig. 7b\&c). Nevertheless, ferrostatin-1 is 
currently not an FDA approved drug with questions surrounding safety. Thus, we tested Lcarnosine, a dipeptide composed of beta-alanine and L-histidine that has the ability to scavenge $\mathrm{LOOH} .{ }^{23,24}$ Similar to ferrostatin-1, L-carnosine was sufficient to rescue $\mathrm{LOOH}$ and cell death induced by GPx4 KD (Extended Data Fig. 7d-f) or erastin (Extended Data Fig. 7g-i). Leveraging these data, we performed a preclinical trial for L-carnosine provided in drinking water ad lib $(80 \mathrm{mM})$ in young wildtype C57BL6/J mice. L-carnosine treatment did not alter body mass, body composition, food intake, and water intake (Extended Data Fig. 7j-m), and successfully suppressed muscle LOOH induced by HU (Extended Data Fig. 7n\&o). Remarkably, mice provided with L-carnosine were partly protected from disuseinduced muscle atrophy (Extended Data Fig. 7p). In humans, L-carnosine is rapidly degraded by a circulating carnosinase ${ }^{25}$ that may render oral carnosine treatment ineffective. In contrast, $\mathrm{N}$-acetylcarnosine has a longer half-life and may be a more effective reagent in humans thus improving its translational potential. Similar to ferrostatin-1 and L-carnosine, $\mathrm{N}$-acetylcarnosine also prevented LOOH and cell death induced by GPx4 KD (Extended Data Fig. 8a-c) or erastin (Extended Data Fig. 8d-f). Leveraging these findings, we proceeded with a preclinical trial for $\mathrm{N}$ acetylcarnosine in drinking water ( $80 \mathrm{mM}$, Fig. 3i) in young (4 months, C57BL6/J; Jax colony) and old (20 months, C57BL/6; NIA rodent colony) wildtype mice. Similar to Lcarnosine treatment, $\mathrm{N}$-acetylcarnosine did not alter body mass, body composition, food intake, or water intake (Extended Data Fig. 8g-k), and successfully suppressed muscle $\mathrm{LOOH}$ (Fig. 3j and Extended Data Fig. 8I\&m). Strikingly, similar to our findings in GPx4Tg mice, N-acetylcarnosine ameliorated muscle atrophy (Fig. 3k and Extended Data Fig. 8n) and weakness (Fig. 3l and Extended Data Fig. 8o-q) in both young and old mice. Protection from muscle atrophy was similarly explained by greater myofiber cross-sectional area regardless of fiber-type (Fig. 3m\&n and Extended Data Fig. 8r\&s). These promising observations inform a potential clinical trial to test the efficacy of $\mathrm{N}$-acetylcarnosine treatment in ameliorating muscle atrophy in humans. 

were pretreated with Bafilomycin A1 (BafA1) or MG132 prior to erastin incubation to determine whether $\mathrm{LOOH}$ increases protein degradation in a lysosomal- or proteasomaldependent manner, respectively. Erastin-induced reduction in myotube diameter was suppressed with BafA1, but not with MG132 (Fig. 4a\&b and Extended Data Fig. 9a), suggesting that the lysosome mediates protein degradation by $\mathrm{LOOH} .{ }^{26} \mathrm{We}$ also reproduced these findings with RSL3 treatment (Extended Data Fig. 9b\&c). How does $\mathrm{LOOH}$, a lipid molecule, promote lysosomal-degradation? Upstream of the lysosome, autophagosome formation is mediated by a lipidation of LC3 by ATG3 ${ }^{27}$ Thus, we hypothesized that LOOH may affect the lipidation of LC3. Indeed, GPx4 KD drastically reduced the protein content of p62, LC3-I and LC3-II (Fig. 4c and Extended Data Fig. 9d-h), potentially suggesting that $\mathrm{LOOH}$ may accelerate lysosomal degradation by affecting LC3 lipidation. To test this possibility, we performed a targeted deletion of ATG3 in vitro. Indeed, ATG3 KD completely rescued the reduction in myotube diameter induced by GPx4 KD (Fig. 4d\&e and Extended Data Fig. 9i-I). Leveraging these findings, we generated mice with skeletal muscle-specific tamoxifen-inducible ATG3 knockout (ATG3-MKO) (Fig. $4 \mathrm{f}$ and Extended Data Fig. 10a-c) and studied them with or without HU (Extended Data Fig. 10d-f). Loss of muscle ATG3 was protective from disuse-induced atrophy (Fig. $4 \mathrm{~g}$ and Extended Data Fig. 10g) and weakness (Fig. 4h and Extended Data Fig. 10h) which can be explained by greater myofiber cross-sectional area (Fig. 4i\&j and Extended Data Fig. 10i). protein degradation to contribute to muscle atrophy. However, further assessment of muscle LOOH illuminated a more complex interaction between the lysosome and $\mathrm{LOOH}^{28,29}$ Unexpectedly, quantification of 4-HNE revealed that inhibition of the autophagylysosome axis by ATG3 deletion or BafA1 was sufficient to inhibit LOOH induced by GPx4 deletion, erastin, or RSL3 (Fig. 4k and Extended Data Fig. 10j-p). These findings suggest 
potential role in mediating protein degradation downstream. Indeed, immunofluorescence experiments revealed that 4-HNE is highly co-localized to LAMP2 (Fig. 4I and Extended Data Fig. 10q), consistent with the notion that the lysosome is necessary for $\mathrm{LOOH}$ propagation. To support this idea, an increase in $\mathrm{LOOH}$ by hydrogen peroxide or

197 carmustine (agents that increase pan oxidative stress without acting on GPx4 directly) was completely inhibited by lysosomal inhibition (Fig. 4n and Extended Fig. 10r-t). Together, these observations suggests that the propagation of $\mathrm{LOOH}$ may be mediated by the lysosome (Fig. 40).

In summary, the current findings demonstrate a novel mechanism that indicate $\mathrm{LOOH}$ as the key downstream molecule by which oxidative stress promotes muscle atrophy and weakness. Skeletal muscle LOOH was robustly upregulated with aging and/or disuse, and genetic or pharmacologic suppression of $\mathrm{LOOH}$ was sufficient to rescue muscle atrophy. In particular, $\mathrm{N}$-acetylcarnosine treatment shows a potent effect in preserving muscle mass and strength with disuse in both young and old mice, informing the potential trial to utilize

207 this compound to ameliorate loss of muscle function in humans. We further provide evidence that the autophagy-lysosome axis is essential for the propagation of $\mathrm{LOOH}$, suggesting a novel interaction between these cellular processes that could be interrogated to ameliorate $\mathrm{LOOH}$-induced damage. 


\section{Methods}

Animal models. GPx4+/- and GPx4Tg mice were generated previously. ${ }^{13,20}$ Conditional

213 GPx4 knockout (GPx4cKO+/+) mice were acquired from Jackson Laboratory (Stock No:

214 027964). ${ }^{15}$ Conditional ATG3 knockout (ATG3cKO+/+) mice were previously described. ${ }^{30}$

$215 \mathrm{GPx} 4 \mathrm{cKO}+/+$ mice or ATG3CKO+/+ mice were then crossed with tamoxifen-inducible,

216 skeletal muscle-specific Cre recombinase (HSA-MerCreMer+/-) mice ${ }^{31}$ to generate

217 GPx4cKO+/+; HSAMerCreMer-/- (control) and GPx4cKO+/+; HSA-MerCreMer+/- (skeletal

218 muscle-specific GPx4 knockout; GPx4-MKO) mice or ATG3cKO+/+; HSAMerCreMer-/-

219 (control) and ATG3cKO+/+; HSA-MerCreMer+/- (ATG3-MKO) mice. Tamoxifen-injected (7.5

$220 \mu \mathrm{g} / \mathrm{g}$ body mass, 5 consecutive days) littermates were used. Mice were maintained on a 12-

221 hour light/12-hour dark cycle in a temperature-controlled room. Body composition

222 measurements were taken immediately before terminal experiments with a Bruker Minispec

223 MQ20 nuclear magnetic resonance (NMR) analyzer (Bruker, Rheinstetten, Germany). All

224 mice were bred onto C57BL/6J background and were born at normal Mendelian ratios. Body

225 mass were measured every day during HU. All protocols were approved by Institutional

226 Animal Care and Use Committees at the University of Utah.

227 Hindlimb unloading. Mice underwent 1, 7, or 14 days of $\mathrm{HU}$ (2 mice/cage) using a 228 previously described protocol ${ }^{11,12}$ based on the traditional Morey-Holton design to study 229 disuse atrophy in rodents. Along with daily monitoring of body mass, food intake was 230 monitored every other day to ensure that the mice did not experience excessive weight loss 231 due to malnutrition or dehydration. Following 1, 7, or 14 days of $\mathrm{HU}$, mice were fasted for 4 $232 \mathrm{~h}$ and given an intraperitoneal injection of $80 \mathrm{mg} / \mathrm{kg}$ ketamine and $10 \mathrm{mg} / \mathrm{kg}$ xylazine, after 233 which tissues were harvested. Extensor digitorum longus (EDL), and soleus (SOL) were carefully dissected for weight measurements.

235 Muscle force generation. Force-generating properties of soleus and EDL muscles were measured as previously described. ${ }^{32,33}$ Briefly, soleus/EDL muscles were sutured at each tendon, and muscles were suspended at optimal length (Lo), which was determined by pulse stimulation. After Lo was identified, muscles were stimulated $(0.35$ seconds, pulse width 0.2 milliseconds) at frequencies ranging from 10 to $200 \mathrm{~Hz}$. Muscle length and mass were measured to quantify cross-sectional area for force normalization.

241 Quantitative reverse transcription PCR. Samples were homogenized in TRIzol reagent 242 (Life Technologies) to extract total RNA. One microgram RNA was reverse-transcribed using 243 an IScript cDNA synthesis kit (Bio-Rad). Reverse transcription PCR (RT-PCR) was 244 performed with the Viia 7 Real-Time PCR System (Life Technologies) using SYBR Green 
reagent (Life Technologies). All data were normalized to ribosomal L32 gene expression and were normalized to the mean of the control group. Primers were based on sequences in public databases.

Western blot. Whole muscle or cells were homogenized, and western blots were performed as previously described. ${ }^{11}$ Protein homogenates were analyzed for abundance of phosphorylated 4-hydroxynonenal (4-HNE; ab48506; Abcam), GPx4 (ab125066, Abcam), actin (A2066, MilliporeSigma), GAPDH (14C10, Cell Signaling Technology), p62 (ab56416, Abcam), LC3B (83506, Cell Signaling Technology).

Mass spectrometry. Oxidolipidomics samples were analyzed on the SCIEX 7500 system coupled with ExionLC (SCIEX, Concord, Canada) using multiple reaction monitoring (MRM) analysis. Mobile phase $\mathrm{A}$ is composed of 93:7 acetonitrile:dichloromethane containing $2 \mathrm{mM}$ ammonium acetate and mobile phase $B$ is composed of 50:50 acetonitrile:water containing $2 \mathrm{mM}$ ammonium acetate. A Phenomenex Luna® $\mathrm{NH} 23 \mu \mathrm{m}$ particle size $(4.6 \times 150 \mathrm{~mm})$ was used for separation and column temperature was kept at $40^{\circ} \mathrm{C}$. The total flow rate is 0.7 $\mathrm{mL} / \mathrm{min}$ with a total run time of 17 -minutes. Samples were extracted using the Bligh \& Dyer method. Lower layer was collected, dried down and resuspended in mobile phase A.

Cell culture. $\mathrm{C} 2 \mathrm{C} 12$ myoblasts were grown and maintained in high-glucose Dulbecco's modified Eagle's medium (DMEM), with 10\% fetal bovine serum (FBS), and $0.1 \%$ penicillin/streptomycin. Once 90 to $100 \%$ confluent, C2C12 cells were differentiated into myotubes with low-glucose DMEM, with I-glutamine and $110 \mathrm{mg} / \mathrm{L}$ sodium pyruvate; supplemented with $2 \%$ horse serum, and $0.1 \%$ penicillin-streptomycin. For experiments with erastin (E7781, MilliporeSigma), Ferrostatin-1 (SML0583, MilliporeSigma), and RSL3 (SML2234, MilliporeSigma), C2C12 myotubes were incubated with either $10 \mu \mathrm{M}$ erastin/10 $\mu \mathrm{M}$ Ferrostatin-1/5 $\mu \mathrm{M}$ RSL3/ or equal-volume DMSO directly dissolved into medium. For experiments with L-carnosine (C9625, MilliporeSigma), and N-acetylcarnosine (18817, Cayman), C2C12 myotubes were incubated with $10 \mathrm{mM}$ of L-carnosine/N-acetylcarnosine directly dissolved into medium.

Lentivirus-mediated knockdown of GPx4/LPCAT3/ATG3 Lentivirus-mediated knockdown of experiments were performed as previously described. ${ }^{12,18,34}$ Vectors were decreased using pLKO.1 lentiviral-RNAi system. Plasmids encoding short hairpin RNA (shRNA) for mouse GPx4 (shGPx4: TRCN0000076552), mouse LPCAT3 (shLPCAT3: TRCN0000121437), and mouse ATG3 (shATG3: TRCN0000247442) were obtained from MilliporeSigma. Packaging vector psPAX2 (ID 12260), envelope vector pMD2.G (ID 12259), and scrambled shRNA plasmid (SC: ID 1864) were obtained from Addgene. HEK293T cells in $10 \mathrm{~cm}$ dishes were 
transfected using $50 \mu \mathrm{L} 0.1 \%$ polyethylenimine, $200 \mu \mathrm{L} 0.15 \mathrm{M}$ sodium chloride, and $500 \mu \mathrm{L}$ Opti-MEM (with HEPES, $2.4 \mathrm{~g} / \mathrm{L}$ sodium bicarbonate, and I-glutamine; Gibco 31985) with $2.66 \mu \mathrm{g}$ of psPAX2, $0.75 \mu \mathrm{g}$ of pMD2.G, and $3 \mu \mathrm{g}$ of either scrambled or GPx4/LPCAT3/ATG3 shRNA plasmids. After 48 hours, growth medium was collected, filtered using $0.22 \mu \mathrm{m}$ vacuum filters, and used to treat undifferentiated $\mathrm{C} 2 \mathrm{C} 12$ cells for 48 hours. To ensure that only cells infected with shRNA vectors were viable, cells were selected with puromycin throughout differentiation.

Measurements of myotube diameter. Images of myotubes were visualized at $\times 20$ magnification using an inverted light microscope and captured with a camera (DP74, Olympus). Myotube diameter was measured for at least 100 myotubes from 5 random fields in each group using ImageJ software. The average diameter per myotube was calculated as the mean of ten short-axis measurements taken along the length of the myotube.

Assessment of cell death. Cell death levels were examined by counting the numbers of cells with trypan blue staining. The cells were trypsinized and stained with $0.2 \%$ trypan blue for $5 \mathrm{~min}$. Stained and non-stained cells were counted under a microscope using a hemocytometer.

Immunofluorescence. C2C12 myotubes were fixed with 4\% paraformaldehyde for $10 \mathrm{~min}$ and permeabilized with $0.2 \%$ Triton $\mathrm{X}-100$ for $15 \mathrm{~min}$. After blocking with bovine serum albumin, immunocytochemistry was performed with anti-HNE (ab48506, Abcam), antilysosome associated membrane protein 2 (Lamp-2) (NB300-591, Novus), and Alexa Fluorconjugated secondary antibodies Alexa Fluor® 568 (ab175472, abcam), Alexa Fluor ${ }^{\circledR} 488$ (ab150073, abcam), and DAPI (D1306, Invitrogen). Images were captured using a 63× 1.4 NA oil immersion objective on a Leica SP5 confocal system (Leica). For an experiment, C2C12 myotubes incubated with erastin with or without pretreatment of BaFA1 (SML1661, MilliporeSigma). Soleus muscles were embedded in optimal cutting temperature (OCT) gel and sectioned at $10 \mu \mathrm{m}$ with a cryostat (Microtome Plus). The sections underwent blocking for $1 \mathrm{hr}$ with M.O.M. mouse IgG Blocking Reagent (Vector Laboratories, MKB-2213), $1 \mathrm{hr}$ with primary antibodies (BA.D5, SC.71, BF.F3 all at 1:100 from DSHB). Sections were then probed with the following secondary antibodies: Alexa Fluor 647 (1:250; Invitrogen, A21242), Alexa Fluor 488 (1:500; Invitrogen, A21121), and Alexa Fluor 555 (1:500; Invitrogen, A21426). Negative stained fibers were considered to be IIx. Slides were imaged with an automated wide-field light microscope (Nikon Corp.) using a 10x objective lens. Cross-sectional area and fiber type composition was then quantified utilizing ImageJ software.

Mitochondrial respiration measurements. Mitochondrial $\mathrm{O}_{2}$ utilization was measured 
using the Oroboros $\mathrm{O}_{2} \mathrm{~K}$ Oxygraphs, as previously described. ${ }^{11,12}$ Isolated mitochondria were added to the oxygraph chambers containing buffer $Z$. Respiration was measured in response to the following substrate concentrations: $0.5 \mathrm{mM}$ malate, $5 \mathrm{mM}$ pyruvate, $2 \mathrm{mM}$ ADP, $10 \mathrm{mM}$ succinate, and $1.5 \mu \mathrm{M}$ FCCP.

317 Mitochondrial $\mathrm{H}_{2} \mathrm{O}_{2}$ measurements. Mitochondrial $\mathrm{H}_{2} \mathrm{O}_{2}$ production was measured using the Horiba Fluoromax-4, as previously described. ${ }^{11,12}$ Briefly, skeletal muscle was minced in mitochondria isolation medium ( $300 \mathrm{mM}$ sucrose, $10 \mathrm{mM}$ HEPES, $1 \mathrm{mM}$ EGTA) and subsequently homogenized using a Teflon glass system. Homogenates were then centrifuged at $800 \mathrm{~g}$ for $10 \mathrm{~min}$, after which the supernatant was taken and centrifuged at $12,000 \mathrm{~g}$ for $10 \mathrm{~min}$. The resulting pellet was carefully resuspended in mitochondria isolation medium. $\mathrm{JH}_{2} \mathrm{O}_{2}$ was measured in buffer $\mathrm{Z}$ (MES potassium salt; $105 \mathrm{mM}, \mathrm{KCl} 30 \mathrm{mM}$, $\mathrm{KH}_{2} \mathrm{PO}_{4} 10 \mathrm{mM}, \mathrm{MgCl}_{2} 5 \mathrm{mM}$, and $\mathrm{BSA} 0.5 \mathrm{mg} / \mathrm{ml}$ ) supplemented with $10 \mu \mathrm{M}$ Amplex UltraRed (Invitrogen) and $20 \mathrm{U} / \mathrm{mL}$ CuZnSOD in the presence of the following substrates: 10 $\mathrm{mM}$ succinate, $100 \mu \mathrm{M}$ 1,3-bis(2-chloroethyl)-1-nitrosourea (BCNU/carmustine), and $1 \mu \mathrm{M}$ auranofin. The appearance of the fluorescent product was measured with excitation/emission at $565 / 600 \mathrm{~nm}$.

Administration of L-carnosine/ $\mathrm{N}$-acetylcarnosine in vivo. Carnosine was administered as previously described (75). Briefly, young (4-month-old) or old (20-month-old) C57BL/6J mice were supplemented with $80 \mathrm{mM}$ carnosine dissolved in drinking water $(\mathrm{pH} \mathrm{7.5)}$ for 2 weeks ( 1 week of pretreatment and 1 week during $\mathrm{HU}$ ). Bottles were refreshed two times a week (L-carnosine, C9625, MilliporeSigma), and or everyday ( $\mathrm{N}$-acetyl carnosine, 18817, Cayman).

MDA quantification. MDA content was quantified in fresh gastrocnemius muscles using a lipid peroxidation assay kit (ab118970, Abcam) according to the manufacturer's instruction.

337 Rates of appearance of MDA-thiobarbituric acid adduct were quantified colorimetrically at $532 \mathrm{~nm}$ using a spectrophotometer.

Statistical analyses. Data are presented as means \pm s.e.m. Statistical analyses were performed using GraphPad Prism 7.03. Independent sample t-tests (two-sided) were used to compare two groups. For multiple comparisons, one- or two-way analysis of variance

342 (ANOVA) were performed followed by appropriate post-hoc tests corrected for multiple comparisons. For all tests $\mathrm{P}<0.05$ was considered statistically significant. 


\section{References}

3451 Scicchitano, B. M., Pelosi, L., Sica, G. \& Musaro, A. The physiopathologic role of oxidative stress in skeletal muscle. Mech Ageing Dev 170, 37-44, doi:10.1016/j.mad.2017.08.009 (2018).

2 Powers, S. K., Smuder, A. J. \& Criswell, D. S. Mechanistic links between oxidative stress and disuse muscle atrophy. Antioxid Redox Signal 15, 2519-2528, doi:10.1089/ars.2011.3973 (2011).

3 Evans, W. J. Skeletal muscle loss: cachexia, sarcopenia, and inactivity. Am J Clin Nutr 91, 1123S-1127S, doi:10.3945/ajcn.2010.28608A (2010).

4 Larsson, L. et al. Sarcopenia: Aging-Related Loss of Muscle Mass and Function. Physiol Rev 99, 427-511, doi:10.1152/physrev.00061.2017 (2019).

5 Wiernicki, B. et al. Excessive phospholipid peroxidation distinguishes ferroptosis from other cell death modes including pyroptosis. Cell Death Dis 11, 922, doi:10.1038/s41419-020-03118-0 (2020).

6 Yang, W. S. et al. Regulation of ferroptotic cancer cell death by GPX4. Cell 156, 317-331, doi:10.1016/j.cell.2013.12.010 (2014).

7 Anderson, E. J. et al. A carnosine analog mitigates metabolic disorders of obesity by reducing carbonyl stress. J Clin Invest 128, 5280-5293, doi:10.1172/JCI94307 (2018).

8 Bhattacharya, A. et al. Denervation induces cytosolic phospholipase A2-mediated fatty acid hydroperoxide generation by muscle mitochondria. J Biol Chem $\mathbf{2 8 4}, 46$ 55, doi:10.1074/jbc.M806311200 (2009).

9 Pharaoh, G. et al. Targeting cPLA2 derived lipid hydroperoxides as a potential intervention for sarcopenia. Sci Rep 10, 13968, doi:10.1038/s41598-020-70792-7 (2020).

10 Kagan, V. E. et al. Oxidized arachidonic and adrenic PEs navigate cells to ferroptosis. Nat Chem Biol 13, 81-90, doi:10.1038/nchembio.2238 (2017).

11 Eshima, H. et al. Neutralizing mitochondrial ROS does not rescue muscle atrophy induced by hindlimb unloading in female mice. J Appl Physiol (1985) 129, 124-132, doi:10.1152/japplphysiol.00456.2019 (2020).

12 Heden, T. D. et al. Mitochondrial PE potentiates respiratory enzymes to amplify skeletal muscle aerobic capacity. Sci Adv 5, eaax8352, doi:10.1126/sciadv.aax8352 (2019).

13 Yant, L. J. et al. The selenoprotein GPX4 is essential for mouse development and protects from radiation and oxidative damage insults. Free Radic Biol Med 34, 496502, doi:10.1016/s0891-5849(02)01360-6 (2003).

14 Katunga, L. A. et al. Obesity in a model of gpx4 haploinsufficiency uncovers a causal role for lipid-derived aldehydes in human metabolic disease and cardiomyopathy. Mol Metab 4, 493-506, doi:10.1016/j.molmet.2015.04.001 (2015).

15 Yoo, S. E. et al. Gpx4 ablation in adult mice results in a lethal phenotype accompanied by neuronal loss in brain. Free Radic Biol Med 52, 1820-1827, doi:10.1016/j.freeradbiomed.2012.02.043 (2012).

16 Imai, H. \& Nakagawa, Y. Biological significance of phospholipid hydroperoxide glutathione peroxidase (PHGPx, GPx4) in mammalian cells. Free Radic Biol Med 34, 145-169, doi:10.1016/s0891-5849(02)01197-8 (2003).

17 Lee, J. Y., Kim, W. K., Bae, K. H., Lee, S. C. \& Lee, E. W. Lipid Metabolism and Ferroptosis. Biology (Basel) 10, doi:10.3390/biology10030184 (2021).

18 Ferrara, P. J. et al. Lysophospholipid acylation modulates plasma membrane lipid organization and insulin sensitivity in skeletal muscle. J Clin Invest 131, doi:10.1172/JCl135963 (2021).

19 Ferrara, P. J. et al. Low lysophosphatidylcholine induces skeletal muscle myopathy that is aggravated by high-fat diet feeding. FASEB $\mathrm{J} 35$, e21867, doi:10.1096/fj.202101104R (2021). 
20 Ran, Q. et al. Transgenic mice overexpressing glutathione peroxidase 4 are protected against oxidative stress-induced apoptosis. J Biol Chem 279, 5513755146, doi:10.1074/jbc.M410387200 (2004).

21 Dixon, S. J. et al. Ferroptosis: an iron-dependent form of nonapoptotic cell death. Cell 149, 1060-1072, doi:10.1016/j.cell.2012.03.042 (2012).

22 Codenotti, S. et al. Cell growth potential drives ferroptosis susceptibility in rhabdomyosarcoma and myoblast cell lines. J Cancer Res Clin Oncol 144, 17171730, doi:10.1007/s00432-018-2699-0 (2018).

23 Cripps, M. J. et al. Carnosine scavenging of glucolipotoxic free radicals enhances insulin secretion and glucose uptake. Sci Rep 7, 13313, doi:10.1038/s41598-01713649-w (2017).

24 Everaert, I., De Naeyer, H., Taes, Y. \& Derave, W. Gene expression of carnosinerelated enzymes and transporters in skeletal muscle. Eur J Appl Physiol 113, 11691179, doi:10.1007/s00421-012-2540-4 (2013).

25 Boldyrev, A. A., Aldini, G. \& Derave, W. Physiology and pathophysiology of carnosine. Physiol Rev 93, 1803-1845, doi:10.1152/physrev.00039.2012 (2013).

$26 \mathrm{Gao}, \mathrm{H}$. et al. Ferroptosis is a lysosomal cell death process. Biochem Biophys Res Commun 503, 1550-1556, doi:10.1016/j.bbrc.2018.07.078 (2018).

27 Ichimura, Y. et al. A ubiquitin-like system mediates protein lipidation. Nature 408, 488-492, doi:10.1038/35044114 (2000).

28 Chen, X., Yu, C., Kang, R., Kroemer, G. \& Tang, D. Cellular degradation systems in ferroptosis. Cell Death Differ 28, 1135-1148, doi:10.1038/s41418-020-00728-1 (2021).

29 Gao, M. et al. Ferroptosis is an autophagic cell death process. Cell Res 26, 10211032, doi:10.1038/cr.2016.95 (2016).

30 Cai, J. et al. Autophagy Ablation in Adipocytes Induces Insulin Resistance and Reveals Roles for Lipid Peroxide and Nrf2 Signaling in Adipose-Liver Crosstalk. Cell Rep 25, 1708-1717 e1705, doi:10.1016/j.celrep.2018.10.040 (2018).

31 McCarthy, J. J., Srikuea, R., Kirby, T. J., Peterson, C. A. \& Esser, K. A. Inducible Cre transgenic mouse strain for skeletal muscle-specific gene targeting. Skelet Muscle 2, 8, doi:10.1186/2044-5040-2-8 (2012).

32 Ferrara, P. J., Verkerke, A. R. P., Brault, J. J. \& Funai, K. Hypothermia Decreases O2 Cost for Ex Vivo Contraction in Mouse Skeletal Muscle. Med Sci Sports Exerc 50, 2015-2023, doi:10.1249/MSS.0000000000001673 (2018).

33 Verkerke, A. R. P. et al. Phospholipid methylation regulates muscle metabolic rate through $\mathrm{Ca}(2+)$ transport efficiency. Nat Metab 1, 876-885, doi:10.1038/s42255019-0111-2 (2019).

34 Johnson, J. M. et al. Alternative splicing of UCP1 by non-cell-autonomous action of PEMT. Mol Metab 31, 55-66, doi:10.1016/j.molmet.2019.10.007 (2020). 
438

439

440

441

442

443

444

445

446

447

448

449

450

451

452

453

454

455

456

457

458

459

460

461

462

\section{Acknowledgements}

This research is supported by NIH DK107397, GM144613, AG063077, University of Utah Center on Aging (to K.F.), AG050781 (to M.J.D.), HL122863, AG057006 (E.J.A), AG064078 (to Q.R.), HL149870 (S.B.), HL139451 (to Z.S.M), DK130555 (to A.D.P.), AG073493 (to J.J.P.), American Heart Association 18PRE33960491 (to A.R.P.V.) and 19PRE34380991 (to J.M.J.), Larry H. \& Gail Miller Family Foundation (to P.J.F.), and Uehara Memorial Foundation (to H.E.). We would like to thank Diana Lim from the University of Utah Molecular Medicine Program for assistance with figures.

\section{Author contributions}

H.E. and K.F. contributed to study concept design and wrote the manuscript. P.S. and J.L.S. contributed to study design and data analysis. M.J.P., J.A.M., and J.E.C. performed mass spectrometry analyses. M.J.D. and Z.S.M. performed human muscle biopsies. H.E., P.S., J.L.S., A.W.L., J.M.J., J.J.P., and A.S., performed mouse experiments. H.E. performed all biochemical assays, body composition measurements and analysis of muscle force production. A.R.P.V. and P.J.F. assisted in muscle functional measurements. P.J.F. assisted in cell culture experiments. E.J.A assisted in L-carnosine and $\mathrm{N}$-acetylcarnosine experiments. S.B. and Q.R. designed and generated mouse models. All contributed to editing the manuscript.

\section{Competing interests}

No competing interests to disclose.

\section{Materials \& Correspondence}

Correspondence and material requests should be addressed to K. Funai. 
Fig. 1 | LOOH increases with aging and disuse in skeletal muscle. a,b GPx4 mRNA levels in skeletal muscle biopsy samples from young and old humans ( $n=9$ for young, $n=$ 18 for old) (a) or skeletal muscles from young and old mice ( $n=8$ for young, $n=7$ for old) (b). c,d, Oxidized phospholipid content in skeletal muscle from young and old mice $(n=8$ per group). e,f, Soleus muscle mass (e), force-frequency curve (f) in soleus muscle following 1 and 7 days of HU from young WT C57BL/6J mice ( $n=4$ for sham, $n=6$ for 1 day HU, $n=$ 6 for 7 day HU). g, Immunoblotting of 4-HNE following 1 and 7 days of HU. h. A schematic on how pathways that regulate $\mathrm{LOOH}$ may promote muscle atrophy. PUFA: phospholipids containing polyunsaturated fatty acids. i,j, Representative images (i), and quantification (j) of myotube diameter ( $n=104$ for scrambled: SC, $n=107$ for GPx4 KD, $n=117$ for Vehicle, $n$ $=120$ for erastin, $n=104$ for Vehicle, $n=110$ for RSL3). Scale bar, $100 \mu \mathrm{m}$. Data are shown as the mean \pm s.e.m. Statistical analyses in $\mathbf{a}, \mathbf{b}, \mathbf{c}, \mathbf{d}$ and $\mathbf{j}$ were performed with an unpaired two-tailed t-test. Statistical analyses in e were performed with a one-way ANOVA with Dunnett's multiple comparisons test. Statistical analyses in $\mathbf{f}$ were performed with a two-way ANOVA and multiple comparisons were performed using Tukey's multiple comparisons tests.

Fig. 2 | Elevated LOOH is sufficient to augment disuse-induced muscle atrophy in young and old mice. a, Soleus muscle mass from young or old WT or GPx4+/- mice with or without $\mathrm{HU}$ ( $n=8-11$ per young group, $n=4-8$ per old mice group). $\mathbf{b}$, Force-frequency curve from old WT or GPx4+/- mice ( $n=4-7$ per group). c, Immunoblotting of 4-HNE from old WT or GPx4+/- mice. d, mRNA levels of GPx4 from young control or GPx4-MKO mice ( $n=4$ per group). e,f, Soleus muscle mass (e) ( $n=7-8$ per group) or force-frequency curve (f) from young control or GPx4-MKO mice ( $n=4-7$ per group). g, Immunoblotting of 4-HNE and GPx4 from young GPx4-MKO. h,i, Representative images of MHC immunofluorescence (h) and muscle fiber CSA by fiber type (i) for soleus muscles in young control or GPx4-MKO mice with $\mathrm{HU}$ ( $n=4$ per group). j, Immunoblotting of $4-\mathrm{HNE}, \mathrm{GPx} 4$ and Actin protein in $\mathrm{C} 2 \mathrm{C} 12$ myotubes with or without GPx4 KD and/or LPCAT3 KD. k,l, Representative images (k), and quantification of myotube diameter (I) from C2C12 myotubes with or without GPx4 KD and/or without LPCAT3 KD ( $\mathrm{n}=104-114$ per group). Scale bar, $100 \mu \mathrm{m}$. Data are shown as the mean \pm s.e.m. Statistical analyses in $\mathbf{d}$ were performed with an unpaired two-tailed t-test. Statistical analyses in I were performed with a one-way ANOVA with Dunnett's multiple comparisons test. Statistical analyses in $\mathbf{a}, \mathbf{c}, \mathbf{e}, \mathbf{f}$, and $\mathbf{i}$ were performed with a two-way ANOVA and multiple comparisons were performed using Tukey's (c,e,f,i) or Sidak's (a) multiple comparisons tests. 
Fig. 3 | Genetic and pharmacologic suppression of LOOH ameliorates muscle atrophy and weakness in young and old mice. a, Soleus muscle mass from young or old WT or GPx4Tg mice with or without HU ( $n=6-11$ per young group, $n=7$ per old group). b. Forcefrequency curve from old WT or GPx4Tg mice ( $n=5-7$ per group). c, Immunoblotting of muscle 4-HNE from old WT or GPx4Tg mice. d,e, Representative images of MHC immunofluorescence (d) and muscle fiber CSA by fiber type (e) for soleus muscles in old WT or GPx4Tg mice with HU ( $n=4-6$ per group). $f$, Immunoblotting of 4-HNE, and actin in C2C12 myotubes with or without GPx4 KD and/or Ferrostatin-1. g,h, Representative images (g), and quantification of myotube diameter (h) from $\mathrm{C} 2 \mathrm{C} 12$ myotubes with $\mathrm{GPx} 4 \mathrm{KD}$ and/or

508 Ferrostatin-1 treatments ( $n=102-110$ per group). Scale bar, $100 \mu \mathrm{m}$. i, Schematic illustration of the protocol for administration of $\mathrm{N}$-acetylcarnosine in vivo. j, Immunoblotting of muscle 4$\mathrm{HNE}$ from $\mathrm{N}$-acetylcarnosine treatment in old mice. $\mathbf{k}$, Soleus muscle mass from young or old mice with or without $\mathrm{N}$-acetylcarnosine treatment ( $n=6-8$ per young group, $n=7$ per old group). I, Force-frequency curve from $\mathrm{N}$-acetylcarnosine study in old mice ( $n=4-5$ per group). $\mathbf{m}, \mathbf{n}$, Representative images of MHC immunofluorescence $(\mathbf{m})$ and muscle fiber CSA by fiber type (n) for soleus muscles ( $n=3-4$ per group) in old mice from the N-acetylcarnosine study. Data are shown as the mean \pm s.e.m. Statistical analyses in $\mathbf{h}$ was performed with a oneway ANOVA with Dunnett's multiple comparisons test, and Statistical analyses in $\mathbf{a}, \mathbf{b}, \mathbf{e}, \mathbf{k}, \mathbf{l}$, and $\mathbf{n}$ were performed with a two-way ANOVA and were performed using Tukey's (b,e,l,n) or Sidak's (a,k) multiple comparisons tests.

Fig. 4 | Autophagy-lysosomal system amplifies LOOH to promote muscle atrophy. a,b,

521 Representative images (a), and quantification of myotube diameter (b) from erastin522 stimulated C2C12 myotubes with proteasomal inhibitor MG132 or lysosomal inhibitor BaFA1.

523 ( $n=21-120$ per group). Scale bar, $100 \mu \mathrm{m}$. c, Autophagic flux analyses immunoblotting for 524 LC3-I, LC3-II, p62, and actin in SC or GPx4 KD C2C12 myotuebs with or without BafA1. d,e,

525 Representative images (d) and quantification of myotube diameter (e) from C2C12 myotubes 526 with or without GPx4 KD and/or ATG3 KD) ( $n=104-121$ per group). $f$, mRNA levels of ATG3 527 ( $n=8-32$ per group) from young control or ATG3-MKO mice. $\mathbf{g}$, Soleus muscle mass from 528 control or ATG3-MKO mice ( $n=10-12$ per group). $\mathbf{h}$. Force-frequency curve from young 529 control or ATG3-MKO mice ( $n=9-12$ per group). $\mathbf{i}, \mathbf{j}$, Representative images of MHC 530 immunofluorescence (i) and muscle fiber CSA by fiber type (j) of soleus muscles in young control or ATG3-MKO mice with HU ( $n=6$ per group). $\mathbf{k}$, Immunoblotting of 4-HNE, GPx4, and actin in $\mathrm{C} 2 \mathrm{C} 12$ myotubes with or without GPx4 KD and/or ATG3 KD. I, Confocal 
533 immunofluorescence of LAMP2 (lysosome) and 4-HNE in erastin-stimulated C2C12

534 myotubes with or without $\mathrm{BaFA1}$. $\mathbf{m}$, Representative images of $\mathrm{H}_{2} \mathrm{O}_{2}$ or $\mathrm{BCNU}$-stimulated

535 C2C12 myotubes with or without BaFA1. Scale bar, $100 \mu \mathrm{m}$. n, Immunoblotting of 4-HNE 536 and actin in $\mathrm{H}_{2} \mathrm{O}_{2}$-stimulated $\mathrm{C}_{2} \mathrm{C} 12$ myotubes with or without BafA1. O, A novel role of 537 lysosome in amplifying $\mathrm{LOOH}$. Data are shown as the mean \pm s.e.m. Statistical analyses in 538 f were performed with an unpaired two-tailed t-test. Statistical analyses in $\mathbf{b}, \mathbf{e}$ was performed 539 with a one-way ANOVA with Dunnett's multiple comparisons test, and $\mathbf{g}, \mathbf{h}$, and $\mathbf{j}$ were 540 performed with a two-way ANOVA and multiple comparisons were performed using Tukey's 541 multiple comparisons tests. 
542 Extended Data Fig. 1 (Related to age or disuse experiments in Figure 1). (a) Immunoblotting 543 of 4-HNE and GPx4 in skeletal muscle from young and old C57BL6/J mice. (b,c) 544 Quantification of 4- HNE (b) and MDA levels (c) ( $n=5$ per group) in young and old C57BL6/J 545 mice. (d-f) body mass (d), body composition (e), food intake (f), following 1 , and 7 days of $546 \mathrm{HU}$ from young WT C57BL/6J mice ( $n=4$ for sham, $n=6$ for 1 day HU, $n=6$ for 7day HU).

547 (g) Quantification of 4- HNE ( $n=3$ per group). (h) Rates of oxygen consumption measured 548 in isolated skeletal muscle mitochondria with Krebs cycle substrates ( $n=4$ for sham, $n=5$ 549 for 1 day HU, $n=5$ for 7 day HU). ADP, adenosine diphosphate; FCCP, carbonyl cyanide-p550 trifluoromethoxyphenylhydrazone; Mal, malate; Pyr, pyruvate. (i) Rate of mitochondrial $\mathrm{H}_{2} \mathrm{O}_{2}$ 551 production in response to succinate or Aurorafin/BCNU ( $n=4$ for sham, $n=5$ for 1 day HU, $552 \mathrm{n}=5$ for 7 day HU). BCNU, 1,3-bis[2-chloroethyl]-1-nitrosourea. (j) Percentage of electron 553 leak in muscle mitochondria ( $n=4$ for sham, $n=5$ for 1 day $\mathrm{HU}, \mathrm{n}=5$ for 7 day HU). Data 554 are shown as the mean \pm s.e.m. Statistical analyses in $\mathbf{b}$ and $\mathbf{c}$ were performed with an 555 unpaired two-tailed t-test. Statistical analyses in $\mathbf{d}, \mathbf{e}, \mathbf{f}, \mathbf{g}$, and $\mathbf{j}$ were performed with an one556 way ANOVA and Dunnett's multiple comparisons tests. Statistical analyses in $\mathbf{h}$, and $\mathbf{i}$ were 557 performed with a two-way ANOVA and Tukey's multiple comparison test.

Extended Data Fig. 2 (Related to in vitro experiments in Figure 1). (a) GPx4 mRNA levels in $\mathrm{C} 2 \mathrm{C} 12$ myotubes with or without GPx4 knockdown (GPx4 KD) ( $n=6$ per group). (b-d) Immunoblotting of 4-HNE, GPx4, and actin (b), quantification of 4-HNE (c) proteins and MDA levels (d) ( $n=3$ per group). (e) mRNA levels for CHAC1 and PTGS2 ( $=6$ per group). (f) cell death levels in GPx4 KD myoblast or myotubes ( $n=3$ independent repeats). (g-k) Quantification of 4-HNE (g), immunoblotting of 4-HNE (h) proteins, MDA levels (i), mRNA levels for CHAC1 and PTGS2 (j), and cell death levels $(\mathbf{k})$ in erastin-stimulated $\mathrm{C}_{2} \mathrm{C} 12$ myotubes ( $\mathrm{n}=3$ independent repeats). (I-o) mRNA levels of GPx4 (I), immunoblotting of GPx4 and actin (m), quantification of GPx4 (n), CHAC1 and PTGS2 (o) in RSL3-stimulated $\mathrm{C} 2 \mathrm{C} 12$ myotubes ( $\mathrm{n}=6$ per group). Data are shown as the mean \pm s.e.m. Statistical analyses in $\mathbf{a}, \mathbf{c}, \mathbf{d}, \mathbf{f}, \mathbf{i}, \mathbf{j}, \mathbf{k}, \mathbf{l}, \mathbf{n}$ and $\mathbf{o}$ were performed with an unpaired two-tailed t-test. Statistical analyses in $\mathbf{g}$ were performed with a one-way ANOVA and Dunnett's multiple comparisons tests. Statistical analyses in e were performed with a two-way ANOVA and Tukey's multiple comparison test. old WT or GPx4+/- mice. (b,c) body composition from young ( $n=9$ for WT sham, $n=8$ for 
GPx4 +/- sham, $\mathrm{n}=7$ for WT HU, $\mathrm{n}=6$ for GPx4 +/- HU) (b) and old ( $\mathrm{n}=4$ for WT sham, $\mathrm{n}$

$577=5$ for GPx4 +/- sham, $n=8$ for WT HU, $n=6$ for GPx4 +/- HU) (c) WT or GPx4+/- mice. (d)

578 Food intake during hindlimb unloading $(\mathrm{HU})$ in young $(\mathrm{n}=7$ for WT, $\mathrm{n}=6$ for GPx4 $+/-$ ) and

579 old ( $n=8$ for WT, $n=6$ for GPx4 +/-) WT or GPx4+/- mice. (e) EDL muscle mass from young

580 ( $\mathrm{n}=9$ for WT sham, $\mathrm{n}=8$ for GPx4 +/- sham, $\mathrm{n}=7$ for WT HU, $\mathrm{n}=6$ for GPx4 +/- HU) and

581 old ( $n=9$ for WT sham, $n=8$ for GPx4 +/- sham, $n=7$ for WT HU, $n=6$ for GPx4 +/- HU)

582 WT or GPx4+/- mice. (f) Quantification of muscle 4-HNE from old mice ( $n=3$ per group). (g)

583 MDA levels from old mice ( $n=3$ per group). (h) Force-frequency curve from soleus muscle 584 from young WT or GPx4+/- mice ( $n=5$ for WT sham, $n=5$ for GPx4 +/- sham, $n=7$ for WT $585 \mathrm{HU}, \mathrm{n}=5$ for GPx4 +/- HU). (i,j) Force-frequency curve in EDL muscle from young $(\mathrm{n}=5$ for 586 WT sham, $\mathrm{n}=5$ for GPx4 +/- sham, $\mathrm{n}=7$ for WT HU, $\mathrm{n}=5$ for GPx4 +/- HU) (i) and old ( $\mathrm{n}=$ 5873 for WT sham, $n=4$ for GPx4 +/- sham, $n=6$ for WT HU, $n=6$ for GPx4 +/- HU) (j) WT or $588 \mathrm{GPx} 4+/-$ mice. Data are shown as the mean \pm s.e.m. Statistical analyses in a-j were 589 performed with a two-way ANOVA and using Tukey's multiple comparisons tests.

591 Extended Data Fig. 4 (Related to GPx4-MKO mice in Figure 2). (a) Genotyping gel for 592 GPx4cKO+/+ mice crossed with HSA-MCM+/- mice to yield GPx4cKO+/+; HSA-MCM+/593 (GPx4-MKO) mice. Control littermates (GPx4cKO +/+; HSA-MCM -/-) were used for 594 experiments. Body mass (b), and body composition (c) ( $n=7$ for Ctrl sham, $n=4$ for GPx4595 MKO sham, $n=7$ for Ctrl HU, $n=8$ for GPx4-MKO HU). (d) Food intake during HU ( $n=7$ for 596 Ctrl, $n=8$ for GPx4-MKO). (e,f) EDL muscle mass ( $n=6$ for Ctrl sham, $n=4$ for GPx4-MKO 597 sham, $n=7$ for Ctrl HU, $n=8$ for GPx4-MKO HU) (e) and force-frequency curve from young 598 mice ( $n=6$ for Ctrl sham, $n=4$ for GPx4-MKO sham, $n=6$ for Ctrl HU, $n=7$ for GPx4-MKO $599 \mathrm{HU})(\mathbf{f})$. (g) Quantification of western blotting for 4-HNE from young mice ( $\mathrm{g}=3$ per group). 600 (h) mRNA levels of CHAC1 and PTGS2 in skeletal muscles ( $n=4$ per group). (i,j) Mean 601 muscle fiber CSA (i) and fiber type composition (j) for soleus muscles following HU ( $n=4$ 602 per group). Data are shown as the mean \pm s.e.m. Statistical analyses in $\mathbf{d}$, and $\mathbf{i}$, were 603 performed with an unpaired two-tailed t-test. Statistical analyses in $\mathbf{b}, \mathbf{c}, \mathbf{e}, \mathbf{f}, \mathbf{g}, \mathbf{h}$ and $\mathbf{j}$ were 604 performed with a two-way ANOVA and Tukey's multiple comparison test.

605

606 Extended Data Fig. 5 (Related to LPCAT3 KD studies in Figure 2). (a) mRNA levels of 607 LPCAT3 in C2C12 myotubes with or without LPCAT3 KD ( $\mathrm{n}=3$ per group). (b) mRNA levels 608 of GPx4 and LPCAT3 in C2C12 myotubes with double knockdown for GPx4 and LPCAT3 (n $609=3$ per group). (c) Quantification of 4-HNE proteins in $\mathrm{C} 2 \mathrm{C} 12$ myotubes with or without 
610 LPCAT3 KD and/or GPx4 KD (n=3 per group). (d,e) immunoblotting (d) and quantification

611 (e) of 4-HNE from C2C12 myotubes with or without LPCAT $3 \mathrm{KD}$ and/or erastin $(\mathrm{n}=3$ per

612 group). (f) mRNA levels of LPCAT3 from erastin-stimulated myotubes ( $n=3$ per group). ( $\mathbf{g}, \mathbf{h}$ )

613 Representative images of myotubes $(\mathbf{g})$ and cell death levels $(\mathbf{h})$ from C2C12 myotubes with 614 or without LPCAT3 KD and/or erastin ( $n=3$ independent repeats). Scale bar, $100 \mu \mathrm{m}$. Data 615 are shown as the mean \pm s.e.m. Statistical analyses in $\mathbf{a}, \mathbf{b}$, and $\mathbf{f}$ were performed with an 616 unpaired two-tailed t-test. Statistical analyses in $\mathbf{c}, \mathbf{e}$, and $\mathbf{h}$ were performed with a two-way 617 ANOVA and Tukey's multiple comparison test.

Extended Data Fig. 6 (Related to GPx4Tg mice in Figure 3). (a) Quantification of GPx4 proteins from WT or GPx4Tg with or without $\mathrm{HU}$ ( $\mathrm{n}=3$ per group). (b) Body mass from young ( $n=9$ for WT, $n=8$ for GPx4Tg) and old ( $n=7$ for WT, $n=7$ for GPx4Tg) mice. (c,d) body composition from young mice (c) and old mice (d). (e) Food intake during HU from young and old mice. ( $\mathbf{f}, \mathbf{g})$ EDL muscle mass from young ( $\mathrm{n}=6$ for WT sham, $\mathrm{n}=8$ for GPx4Tg sham, $n=9$ for WT HU, $n=8$ for GPx4Tg HU)(f) and old ( $n=7$ per group) (g) mice. (h) Force-frequency curve in soleus muscle from young mice $(n=5$ for WT sham, $n=6$ for GPx4Tg sham, $n=9$ for WT HU, $n=8$ for GPx4Tg HU). (i,j) Force-frequency curve in EDL muscle from young mice $(n=5$ for WT sham, $n=7$ for GPx4Tg sham, $n=9$ for WT HU, $n=$ 8 for GPx4Tg HU) (i) and old ( $n=5$ for WT sham, $n=5$ for GPx4Tg sham, $n=5$ for WT HU, $\mathrm{n}=6$ for GPx4Tg HU) (j) mice. (k) Quantification of muscle 4-HNE proteins from old mice ( $\mathrm{n}$ = 3 per group). $(\mathbf{I}, \mathbf{m})$ Mean muscle fiber CSA (I) and fiber type composition ( $\mathbf{m})$ for soleus muscles from old mice following $\mathrm{HU}$ ( $n=6$ for WT, $\mathrm{n}=4$ for GPx4Tg). Data are shown as the mean \pm s.e.m. Statistical analyses in I were performed with an unpaired two-tailed t-test. Statistical analyses in a-m were performed with a two-way ANOVA and Tukey's multiple comparison test.

Extended Data Fig. 7 (A pilot L-carnosine study leading to $\mathrm{N}$-acetylcarnosine study in Figure 3). (a-c) Quantification of 4-HNE proteins ( $n=2$ per group) (a), cell death level ( $n=3$ independent repeats) (b), and mRNA levels of CHAC1 and PTGS2 with or without GPx4 KD and/or Ferrostatin-1 ( $n=6$ for SC, $n=5$ for GPx4 KD) (c) in C2C12 myotubes. (d-f) Immunoblotting (d) and quantification (e) of $4-\mathrm{HNE}$, and actin ( $\mathrm{n}=3$ per group) and cell death level ( $n=3$ independent repeats) (f) with or without erastin and/or L-carnosine in C2C12 myotubes. (g-i) Immunoblotting (g) and quantification (h) of $4-\mathrm{HNE}$, and actin ( $\mathrm{n}=3$ per group), and (i) cell death level ( $\mathrm{n}=3$ independent repeats) in $\mathrm{C} 2 \mathrm{C} 12$ myotubes with or without 
C57BL/6J mice with or without HU. Body mass $(j)$, body composition $(n=6$ for Vehicle sham, $n=7$ for L-carnosine sham, $n=12$ for vehicle $\mathrm{HU}, \mathrm{n}=13$ for L-carnosine $\mathrm{HU})(\mathbf{k})$, food intake

647 (I), water intake ( $n=6$ for Vehicle sham, $n=6$ for L-carnosine sham, $n=6$ for vehicle HU, $n$

$648=7$ for L-carnosine $\mathrm{HU})(\mathbf{m})$, immunoblotting (n) and quantification (o) of 4 -HNE $(n=3$ per group), and soleus muscle mass ( $n=6$ for Vehicle sham, $n=7$ for L-carnosine sham, $n=12$ for vehicle $H U, n=13$ for L-carnosine $H U)(p)$. Data are shown as the mean \pm s.e.m. Statistical analyses in $\mathbf{e}$, and $\mathbf{f}$ were performed with a one-way ANOVA and Tukey's multiple comparison test. Statistical analyses in $\mathbf{a}, \mathbf{b}, \mathbf{c}, \mathbf{h}, \mathbf{i}, \mathbf{j}, \mathbf{k}, \mathbf{I}, \mathbf{m}, \mathbf{o}$ and $\mathbf{p}$ were performed with a twoway ANOVA and Tukey's multiple comparison test.

654

Extended Data Fig. 8 (Related to N-acetylcarnosinestudies in Figure 3). (a-c) Immunoblotting (a) and quantification (b) for 4-HNE, GPx4 and actin ( $\mathrm{n}=3$ per group) and cell death level ( $n=3$ independent repeats) (c) from C2C12 myotubes with or without GPx4 $\mathrm{KD}$ and/or N-acetylcarnosine. (d-f) Immunoblotting (d) and quantification (e) of 4-HNE and actin ( $n=3$ per group) and cell death level ( $n=3$ independent repeats) (f) in C2C12 myotubes with or without erastin and/or N-acetylcarnosine. (g-s) Administration of $\mathrm{N}$-acetylcarnosine from young (4-month-old) and old (20-month-old) C57BL/6J mice. Body mass (g), body composition from young mice (h) and old mice (i). Food intake (j), water intake (k), immunoblotting ( $\mathbf{I})$ and quantification $(\mathbf{m})$ of $4-\mathrm{HNE}(\mathrm{n}=3$ per group). ( $\mathbf{n})$ EDL muscle mass from young mice and old mice. (o) Force-frequency curve in soleus muscle from young mice ( $n=5$ for vehicle sham, $n=6$ for $N$-acetylcarnosine sham, $n=5$ for vehicle $H U, n=5$ for $N$ acetylcarnosine $\mathrm{HU})$. $(\mathbf{p}, \mathbf{q})$ Force-frequency curve in EDL muscle from young mice $(n=7$ for vehicle sham, $n=7$ for $\mathrm{N}$-acetylcarnosine sham, $\mathrm{n}=6$ for vehicle $\mathrm{HU}, \mathrm{n}=7$ for $\mathrm{N}$ acetylcarnosine $\mathrm{HU}$ ) (p) and old mice ( $n=6$ for vehicle sham, $n=7$ for $\mathrm{N}$-acetylcarnosine sham, $n=6$ for vehicle $\mathrm{HU}, \mathrm{n}=6$ for $\mathrm{N}$-acetylcarnosine $\mathrm{HU}$ ) (q). (r,s) Average of muscle fiber CSA ( $\mathbf{r}$ ) and fiber type composition (s) for soleus muscles from old mice following HU $(n=3$ for vehicle, $\mathrm{n}=4$ for $\mathrm{N}$-acetylcarnosine). Data are shown as the mean \pm s.e.m. Statistical analyses in $\mathbf{r}$ were performed with an unpaired two-tailed t-test. Statistical analyses in $\mathbf{e}$, and

673 f were performed with a one-way ANOVA and Tukey's multiple comparison test. Statistical 674 analyses in $\mathbf{b}, \mathbf{c}, \mathbf{g}, \mathbf{h}, \mathbf{i}, \mathbf{j}, \mathbf{k}, \mathbf{m}, \mathbf{n}, \mathbf{o}, \mathbf{p}, \mathbf{q}$, and $\mathbf{s}$ were performed with a two-way ANOVA and Tukey's multiple comparison test.

Extended Data Fig. 9 (Related to in vitro experiments in Figure 4). (a) Cell death levels in erastin-stimulated myotubes with MG132 or BaFA1 ( $n=3$ independent repeats). (b,c) 
680

681

682

683

684

685

686

687

688

689

690

691

692

693

694

695

696

697

698

699

700

701

702

703

704

705

706

707

708

709

710

711

712

713

714

( $n=3$ independent repeats). Immunoblotting (d) and quantification (e) of LC3-I, LC3-II, p62 (all static measurements without BafA1), and actin protein from $\mathrm{C} 2 \mathrm{C} 12$ myotubes with or without GPX4 KD ( $n=4$ per group). (f-h) quantification of western blotting for LC3-I (f), LC3II (g), and p62 (h) protein from GPx4 KD myotubes with or without BaFA1 ( $\mathrm{n}=3$ per group). (i) mRNA levels of ATG3 in C2C12 myotubes with or without ( $n=3$ per group). (j) mRNA levels of GPx4 and ATG3 from C2C12 myotubes with double knockdown for ATG3 and GPx4 ( $n=3$ per group). ( $(\mathbf{l}, \mathrm{I})$ Immunoblotting (k) and quantification (I) of LC3, p62, and GPx4 protein from $\mathrm{C} 2 \mathrm{C} 12$ myotubes with or without ATG3 KD ( $n=3$ per group). Data are shown as the mean \pm s.e.m. Statistical analyses in $\mathbf{c}, \mathbf{i}$ and $\mathbf{I}$ were performed with an unpaired two-tailed ttest. Statistical analyses in a were performed with a one-way ANOVA and Tukey's multiple comparison test. Statistical analyses in $\mathbf{e}, \mathbf{f}, \mathbf{g}, \mathbf{h}, \mathbf{i}$, and $\mathbf{j}$ were performed with a two-way ANOVA and Tukey's multiple comparison test.

Extended Data Fig. 10 (Related to in vivo and in vitro experiments in Figure 4). (a) Genotyping of ATG3cKO+/+ mice crossed with HSA-MCM+/- mice to yield ATG3cKO+/+; HSA-MCM+/- (ATG3-MKO) mice. Control littermates (ATG3CKO +/+; HSA-MCM -/-) were used for experiments. (b) Immunoblotting of p62, LC3, and GAPDH proteins from skeletal muscles from control and ATG3-MKO mice. (c) Quantification of LC3-II/I ratio in muscles from control and ATG3-MKO mice ( $\mathrm{n}=8$ per group). (d) Body weight, (e) lean mass, and (f) fat mass from control and ATG3-MKO mice (control sham $n=9$, ATG3-MKO sham $n=10$, control HU $n=10$, ATG3-MKO HU $n=10$ ). (g) EDL muscle mass (control sham $n=10$, ATG3-MKO sham $n=12$, control HU $n=10$, ATG3-MKO HU $n=10$ ). (h) Force frequency curve for EDL muscle (control sham $n=9$, ATG3-MKO sham $n=8$, control HU $n=10$, ATG3MKO HU $n=9$ ). (i) Fiber type composition for SOL muscle (control HU $n=6$, ATG3MKO HU $\mathrm{n}=6$ ). (j) quantification of 4 -HNE protein from $\mathrm{C} 2 \mathrm{C} 12$ myotubes with or without GPx4 KD and/or ATG3 KD ( $\mathrm{n}=3$ per group). (k,I) Immunoblotting (k) and quantification (I) of 4-HNE protein from $\mathrm{C} 2 \mathrm{C} 12$ myotubes with or without erastin and/or ATG3 KD ( $n=3$ per group). $(\mathbf{m}, \mathbf{n})$ Immunoblotting $(\mathbf{m})$ and quantification $(\mathbf{n})$ of $4-\mathrm{HNE}$ protein from $\mathrm{C} 2 \mathrm{C} 12$ myotubes with or without erastin and/or BaFA1 ( $\mathrm{n}=3$ per group). (o,p) Immunoblotting (o) and quantification (p) of 4-HNE protein from C2C12 myotubes with or without RSL3 and/or BaFA1 ( $n=3$ per group). (q) Confocal fluorescence microscope images of erastin-stimulated myotubes with or without BaFA1. Boxed regions are shown enlarged at far right. 4- $\mathrm{HNE}$ protein from $\mathrm{H}_{2} \mathrm{O}_{2}$ stimulated (r), or BCNU-stimulated (s,t) $\mathrm{C} 2 \mathrm{C} 12$ myotubes with or without BafA1. Data are shown as the mean \pm s.e.m. Statistical analyses in $\mathbf{c}, \mathbf{d}, \mathbf{e}, \mathbf{f}, \mathbf{g}, \mathbf{h}, \mathbf{i}, \mathbf{j}, \mathbf{l}, \mathbf{l}, \mathbf{n}, \mathbf{p}, \mathbf{r}$ and $\mathbf{t}$ were performed with a two-way ANOVA and Tukey's multiple comparison test. 

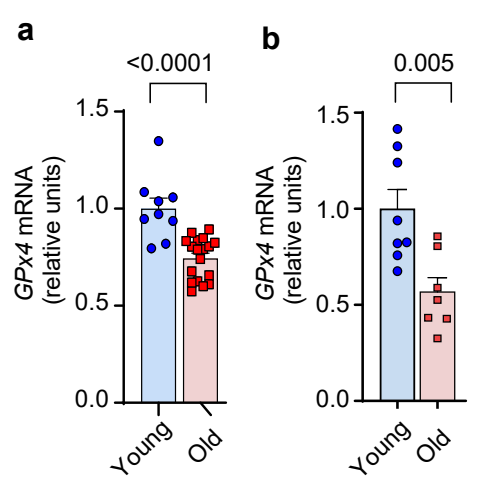

Humans
C

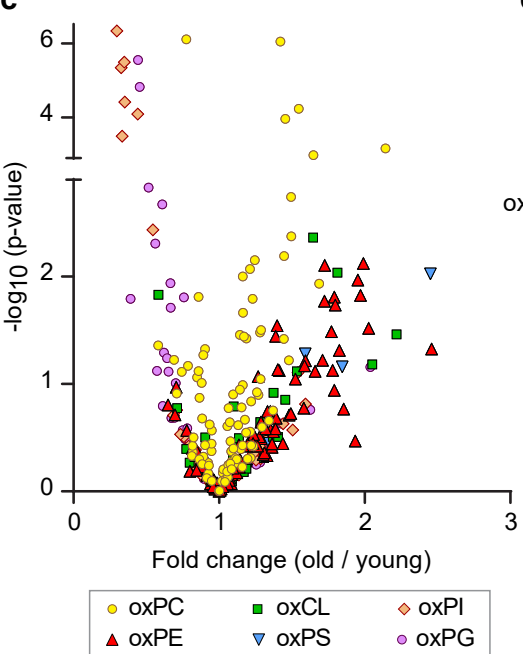

e
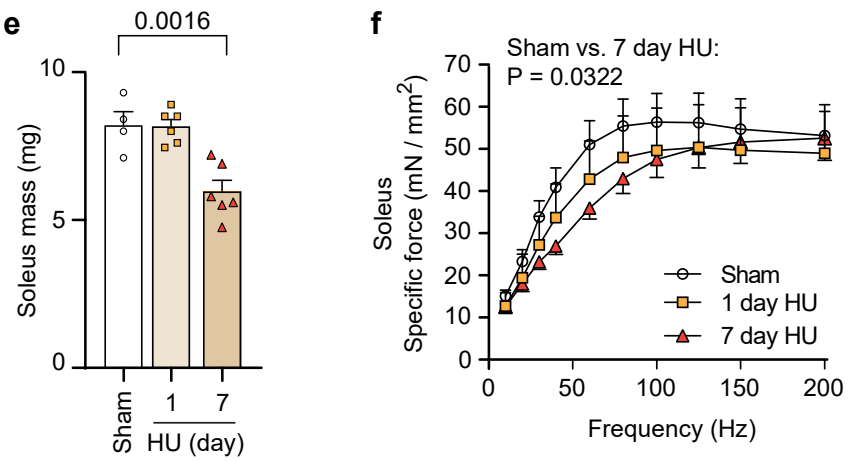

d

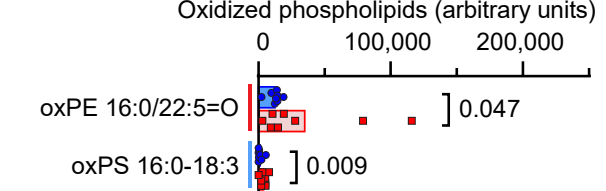

oxCL 18:1-18:1-18:2-20:4 ]0.035

oxPC 16:1/20:5-OH ] 0.0007

OxPE 16:0/22:6-OH ] ] ] 0.030

OXPE 16:1/18:3-OOH | 0.008

OXPE 18:1/22:6-OOH

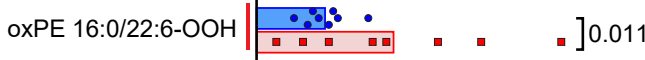

oxPS 18:0-18:1] 0.039

OXPE 16:1/22:5-OOH ] 0.011

- Young

- Old
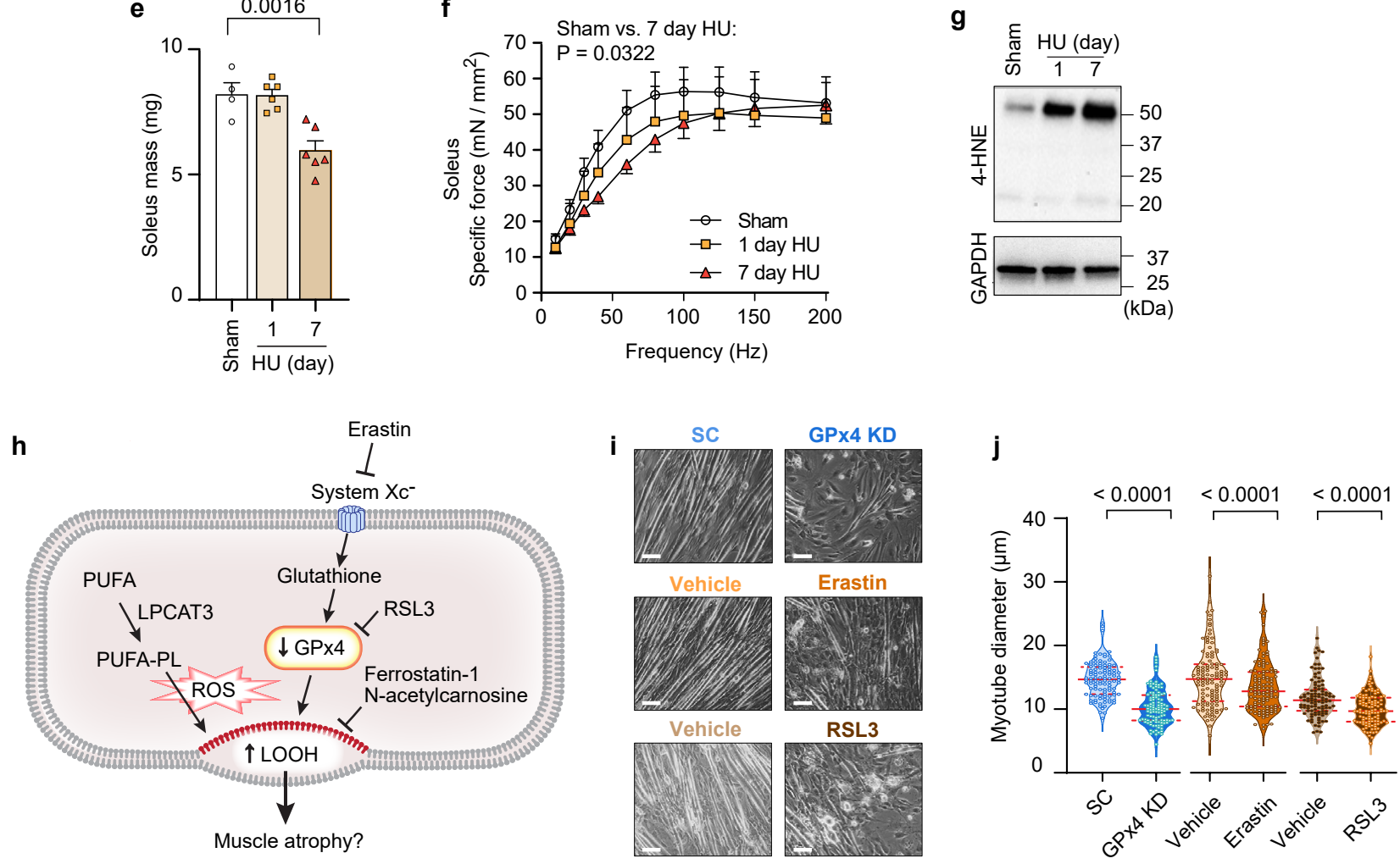

Figure 1 

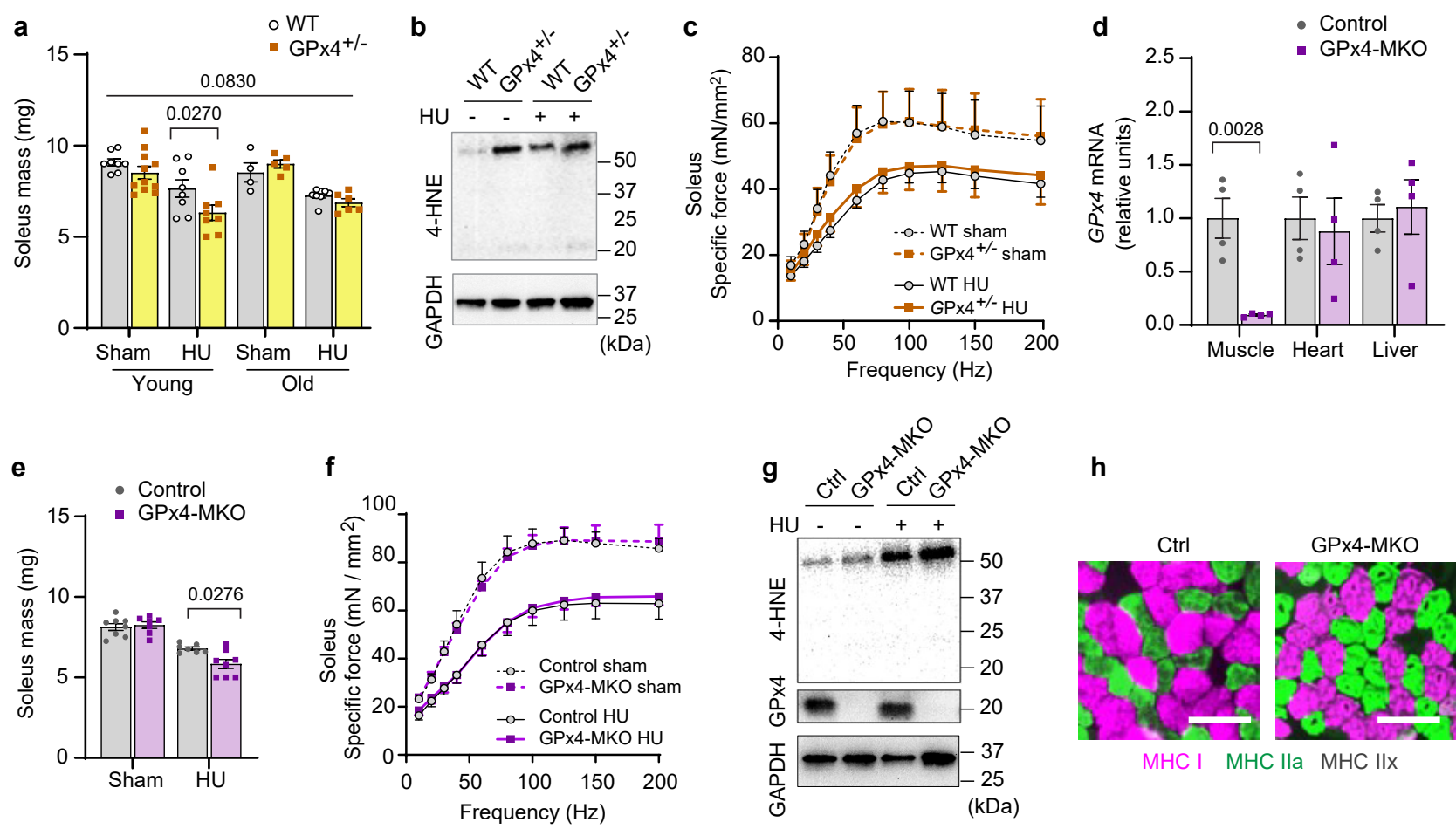

h

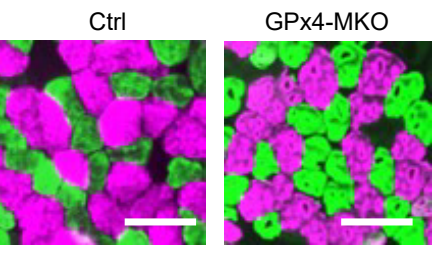

MHC I MHC Ila MHC IIx
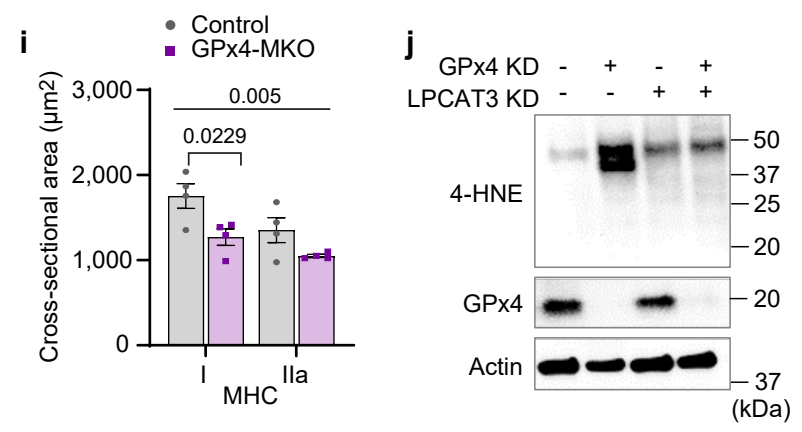

k

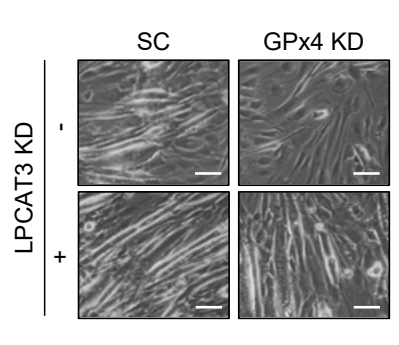

I

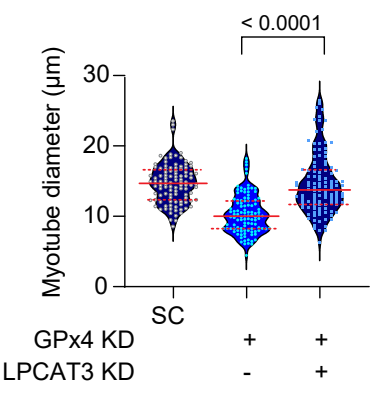

Figure 2 

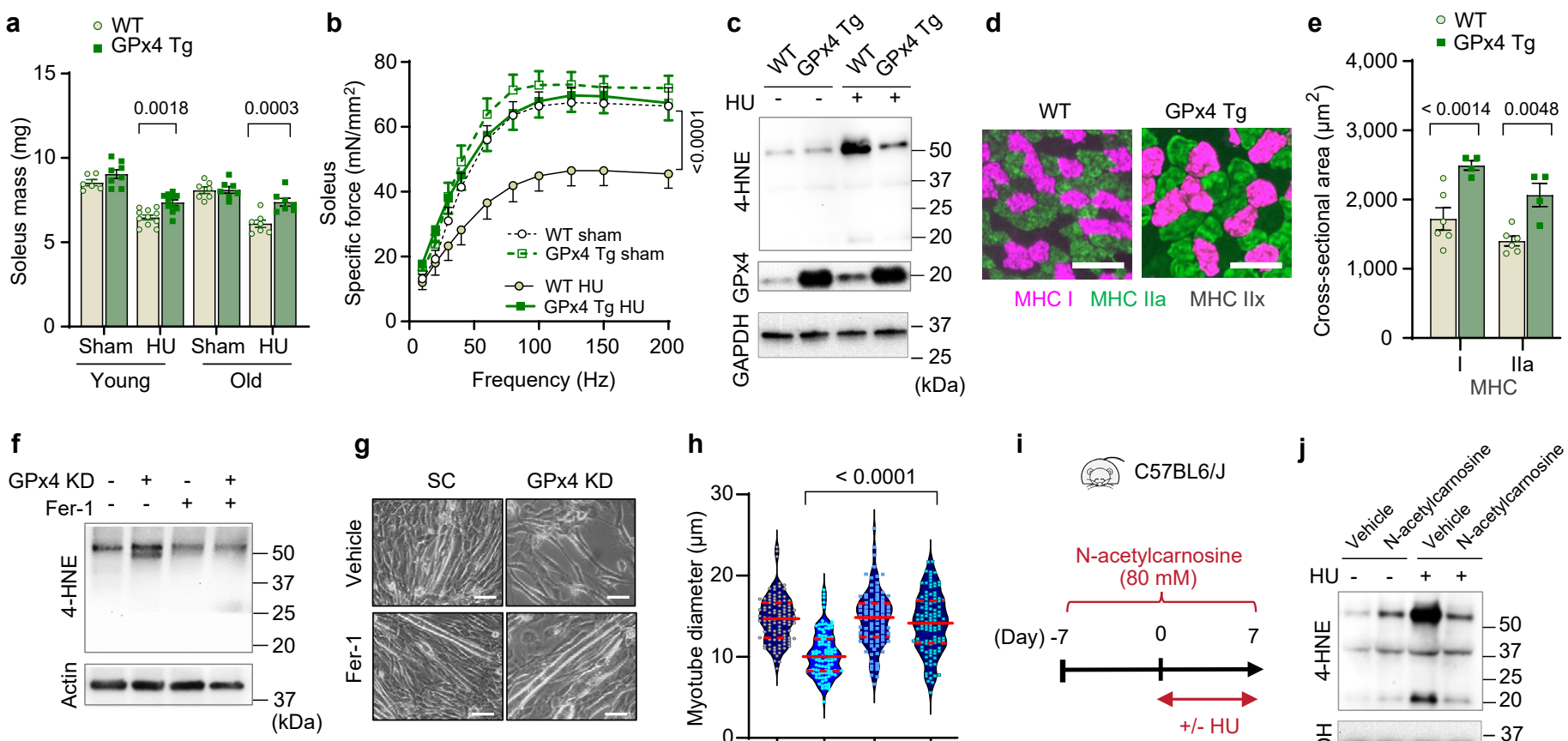

g

h
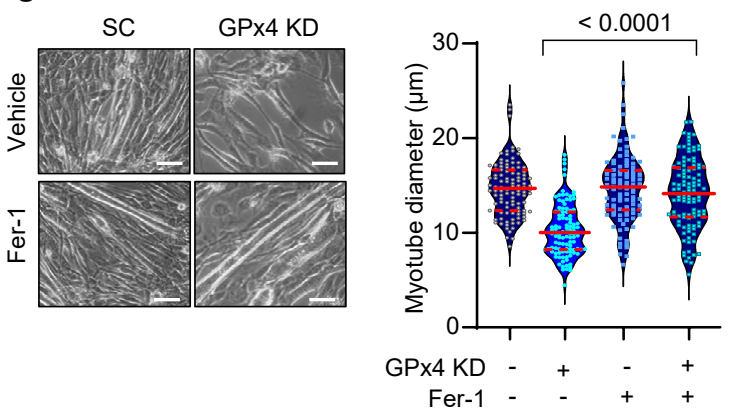

i

T
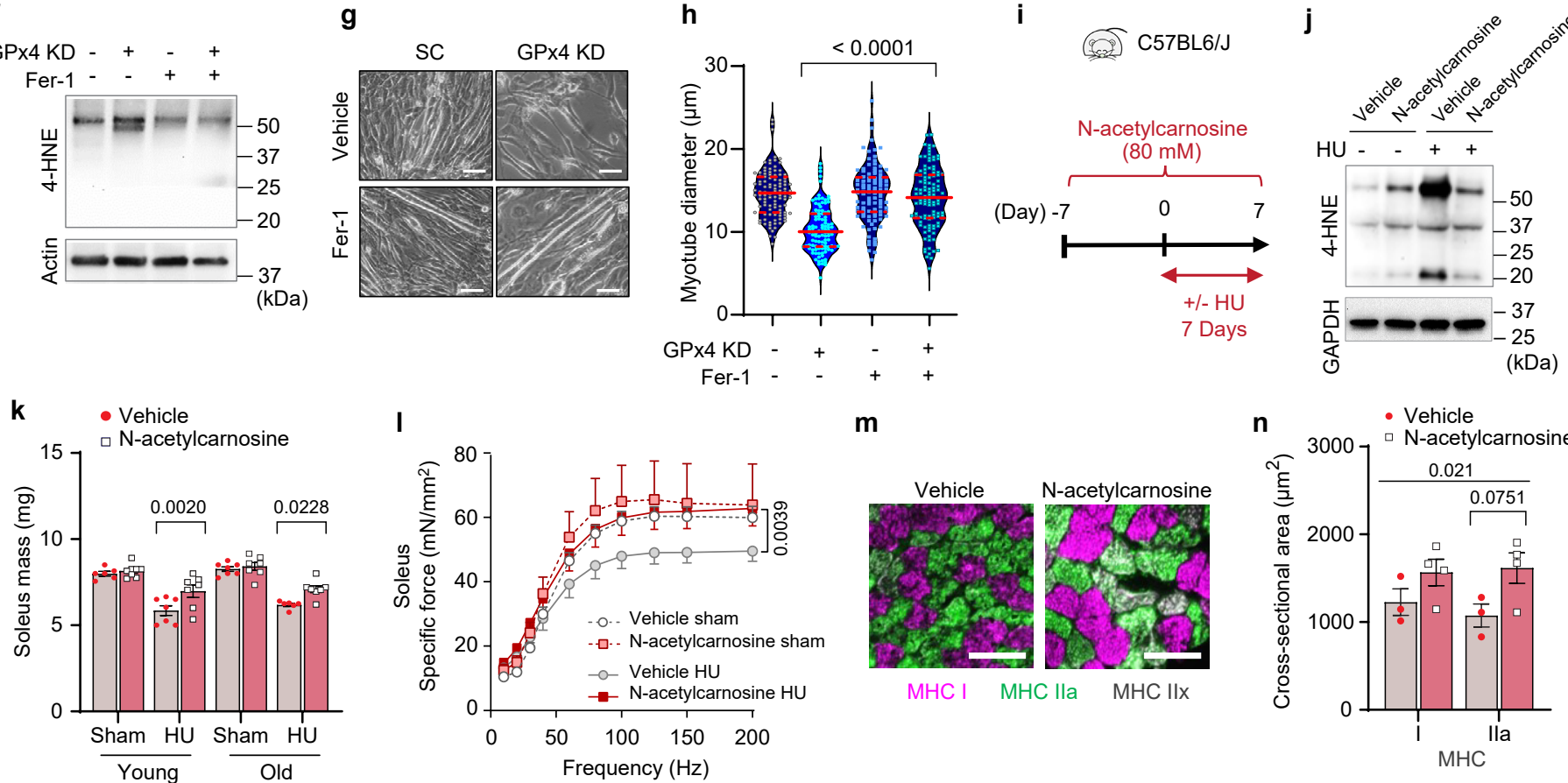

m

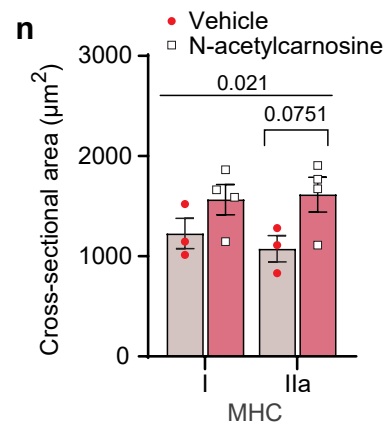

Figure 3 

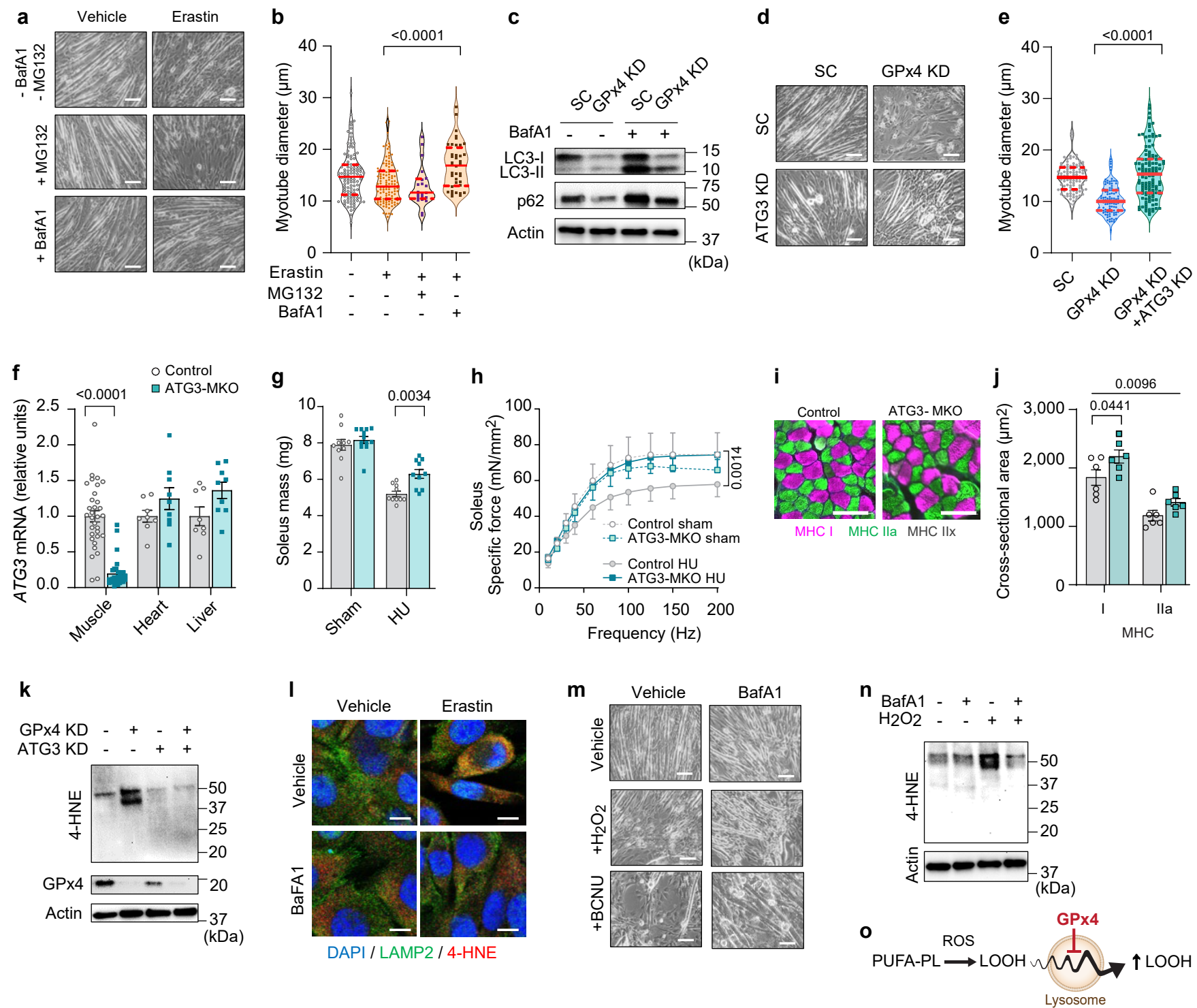

Figure 4 

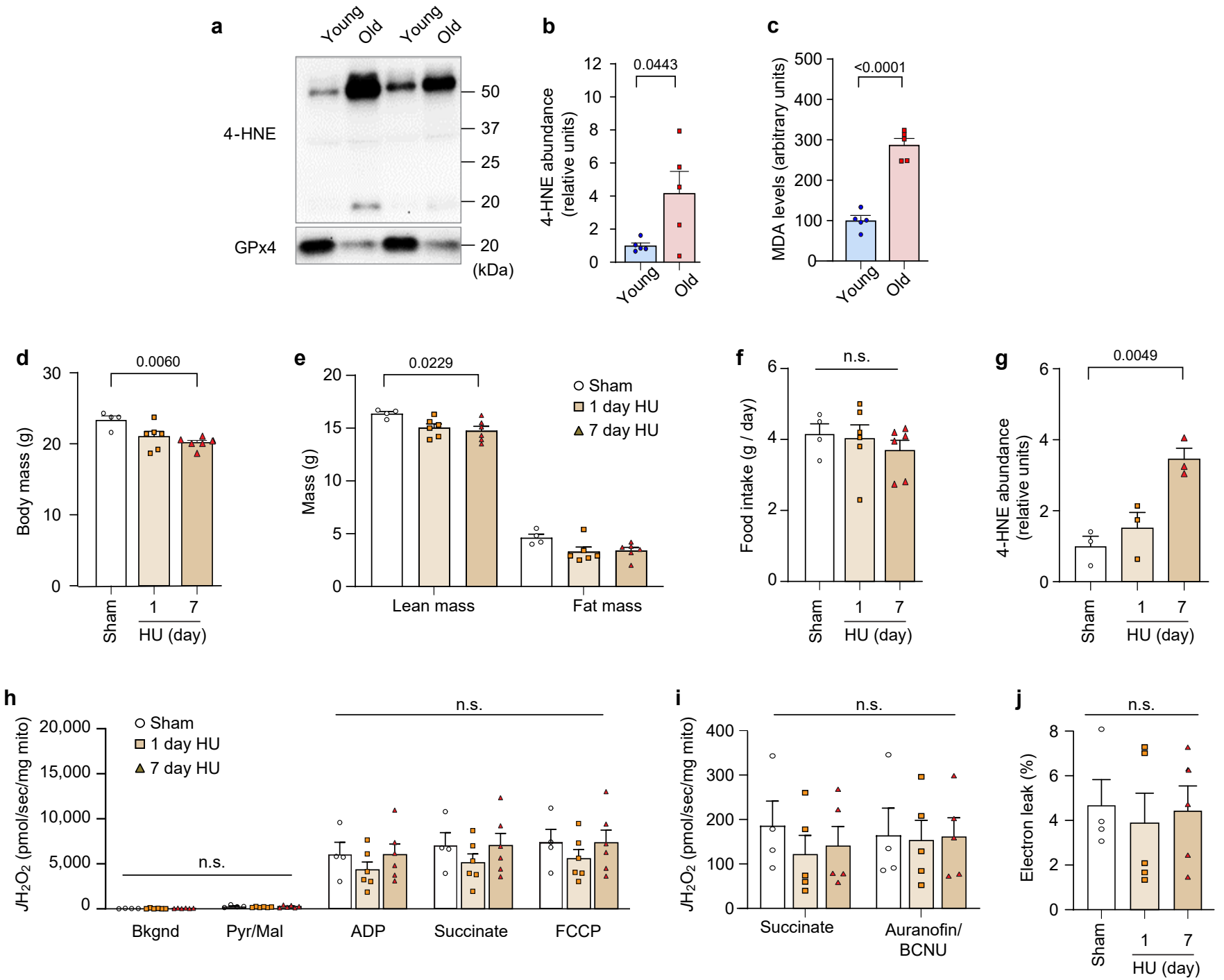

ED 01 
a
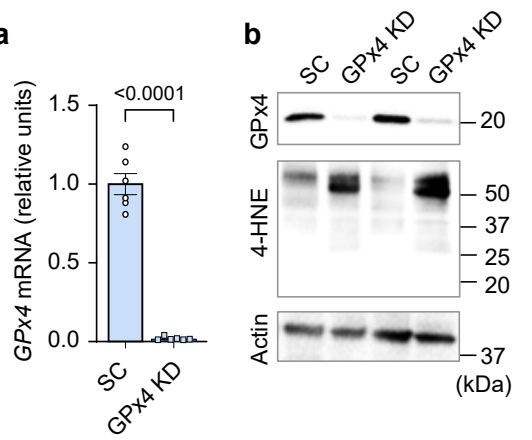

c

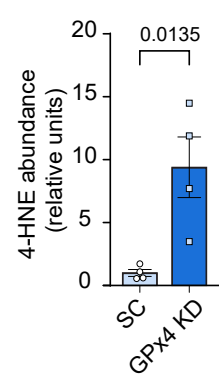

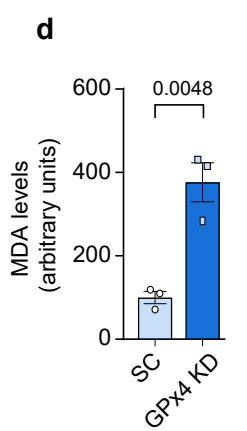

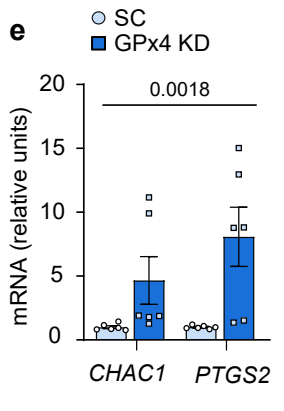

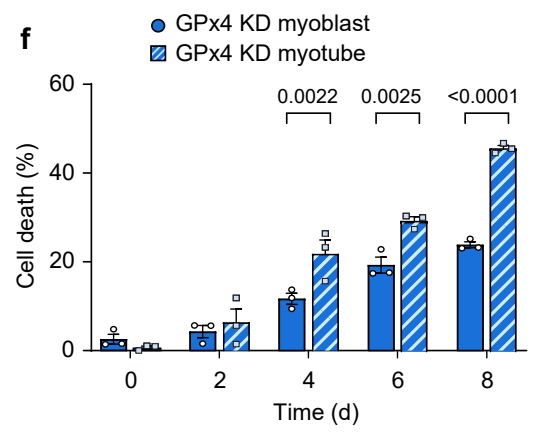
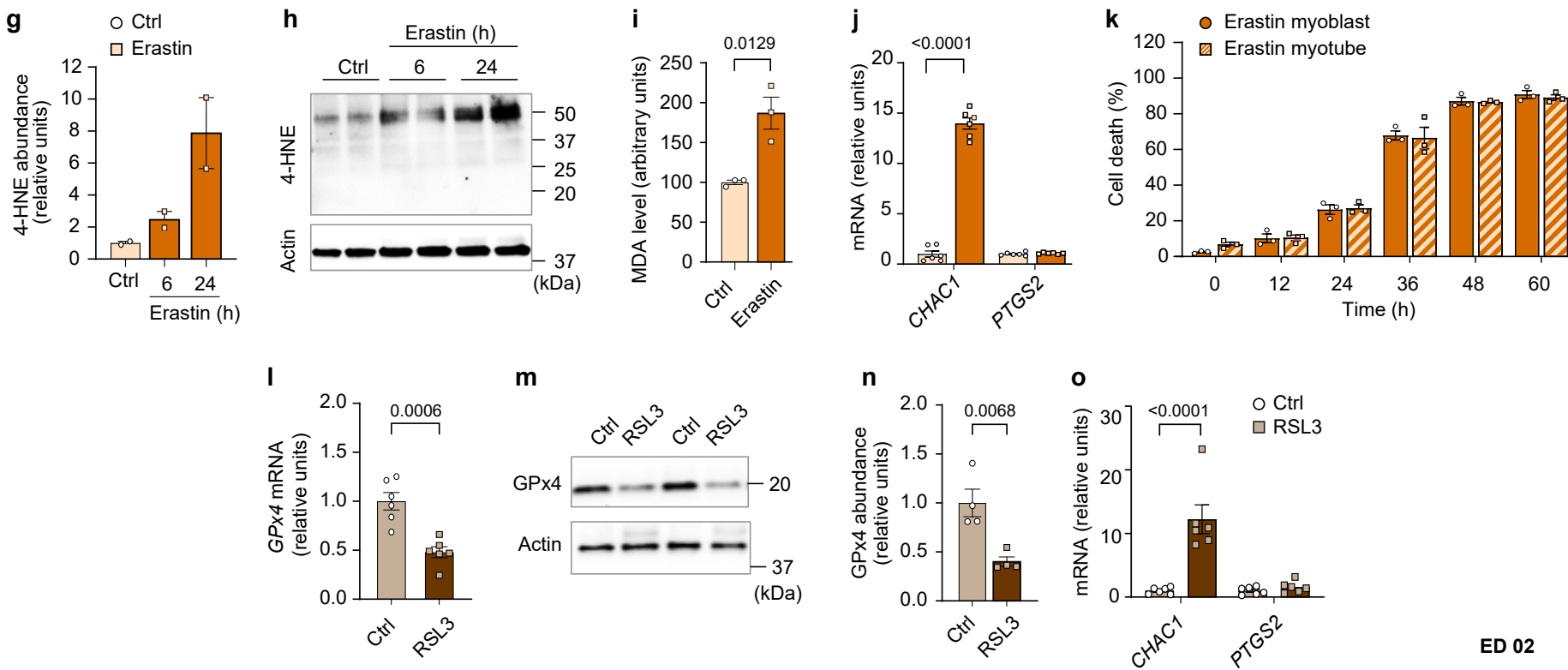

m

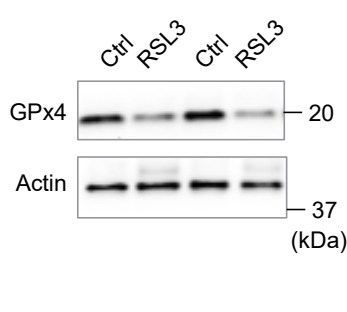

n

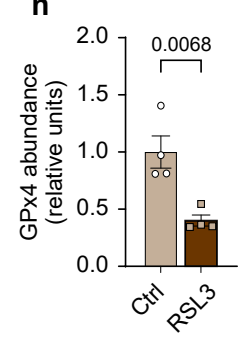

o

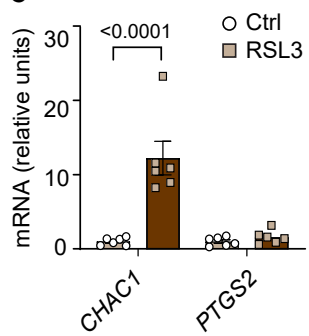

ED 02 

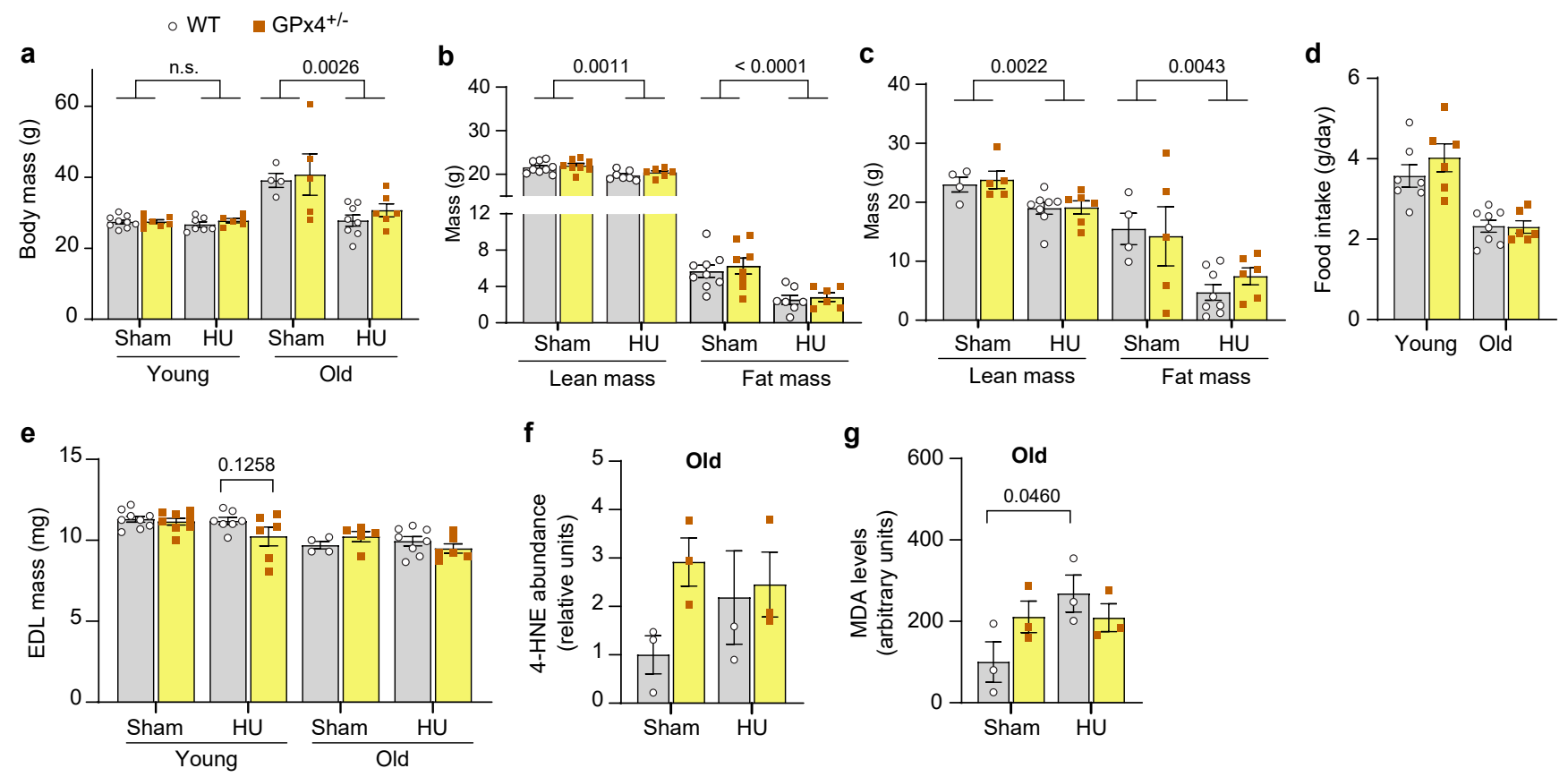

h
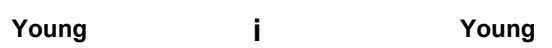

j
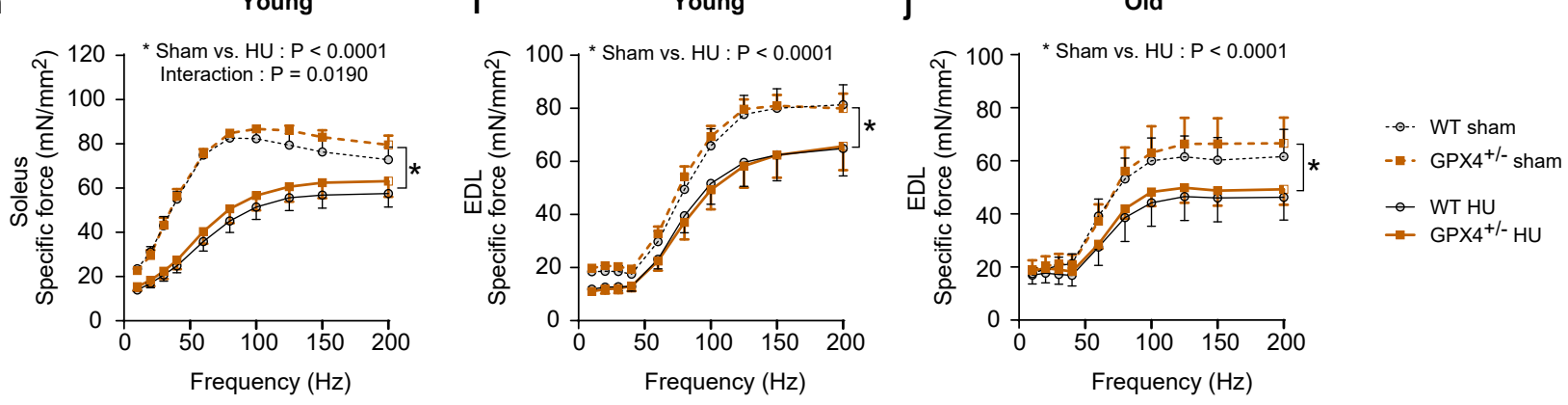

ED 03 

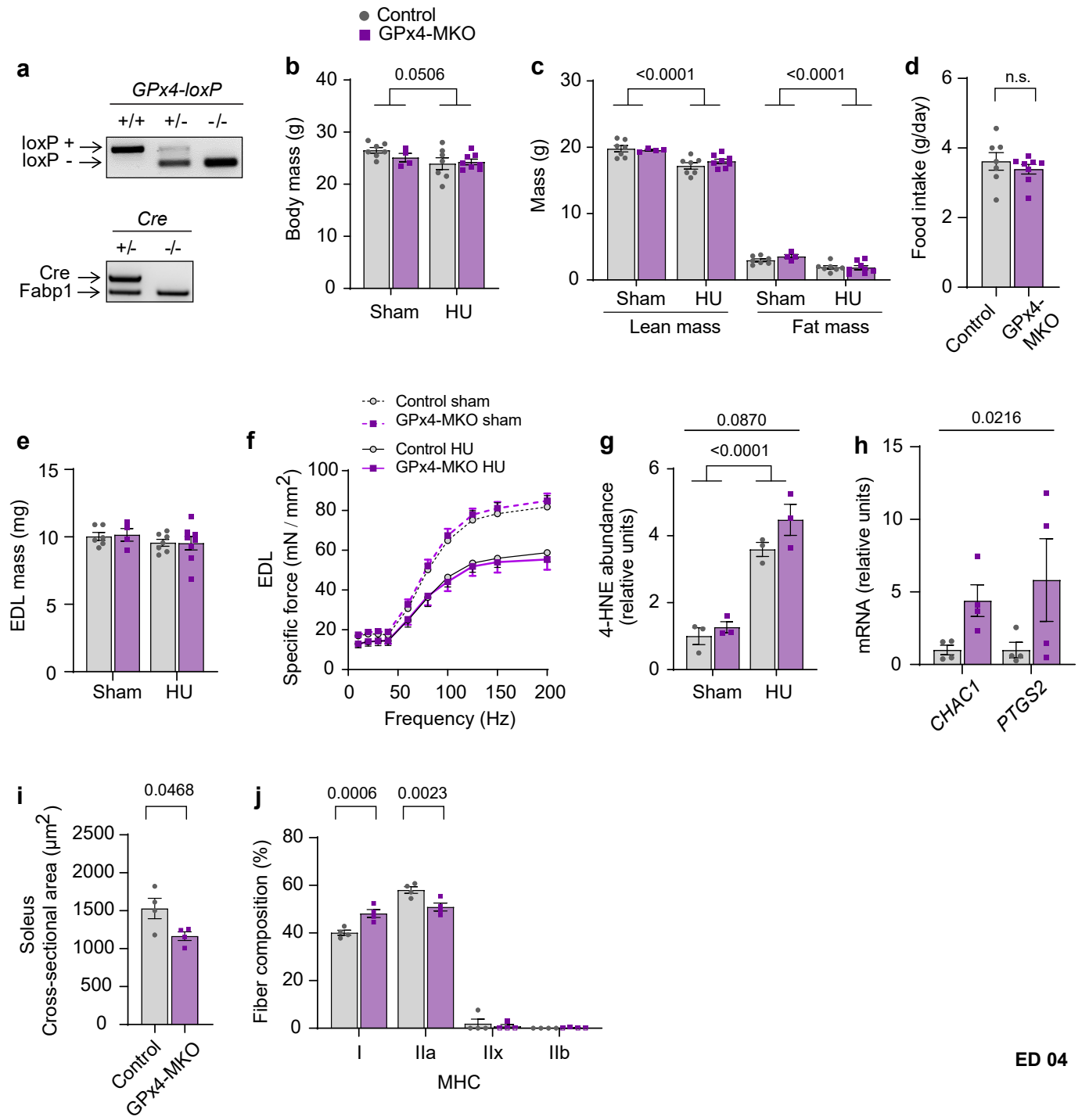

ED 04 


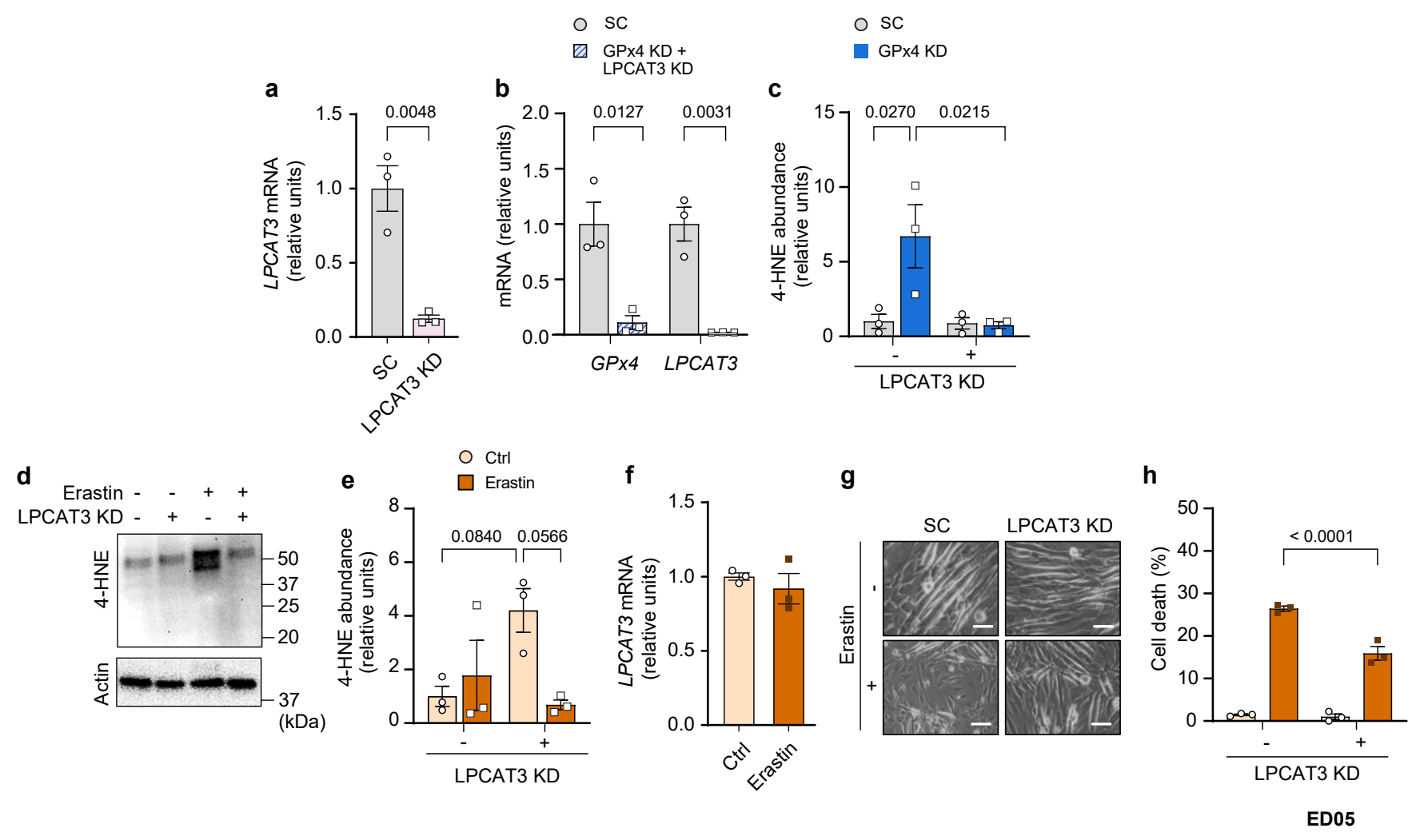



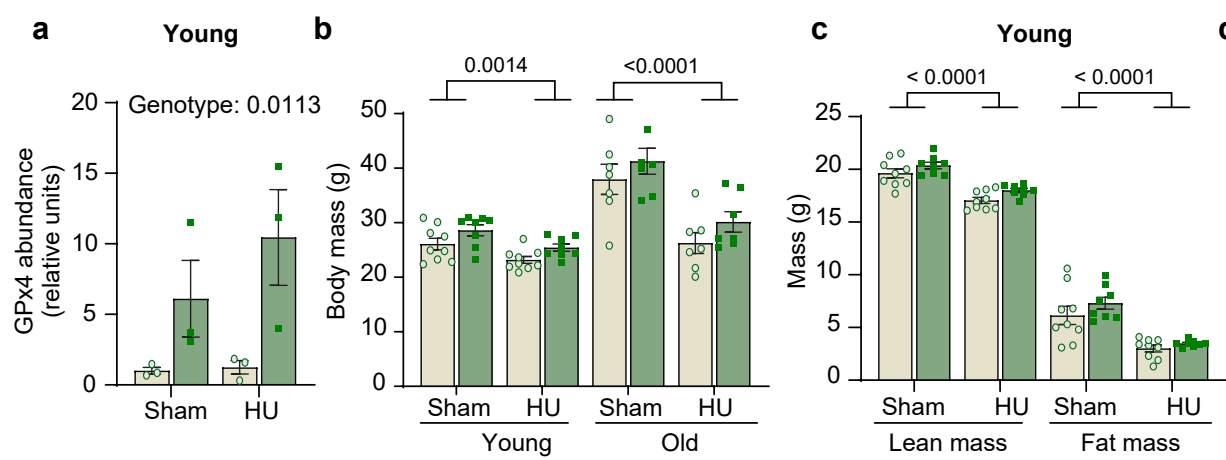

d Old
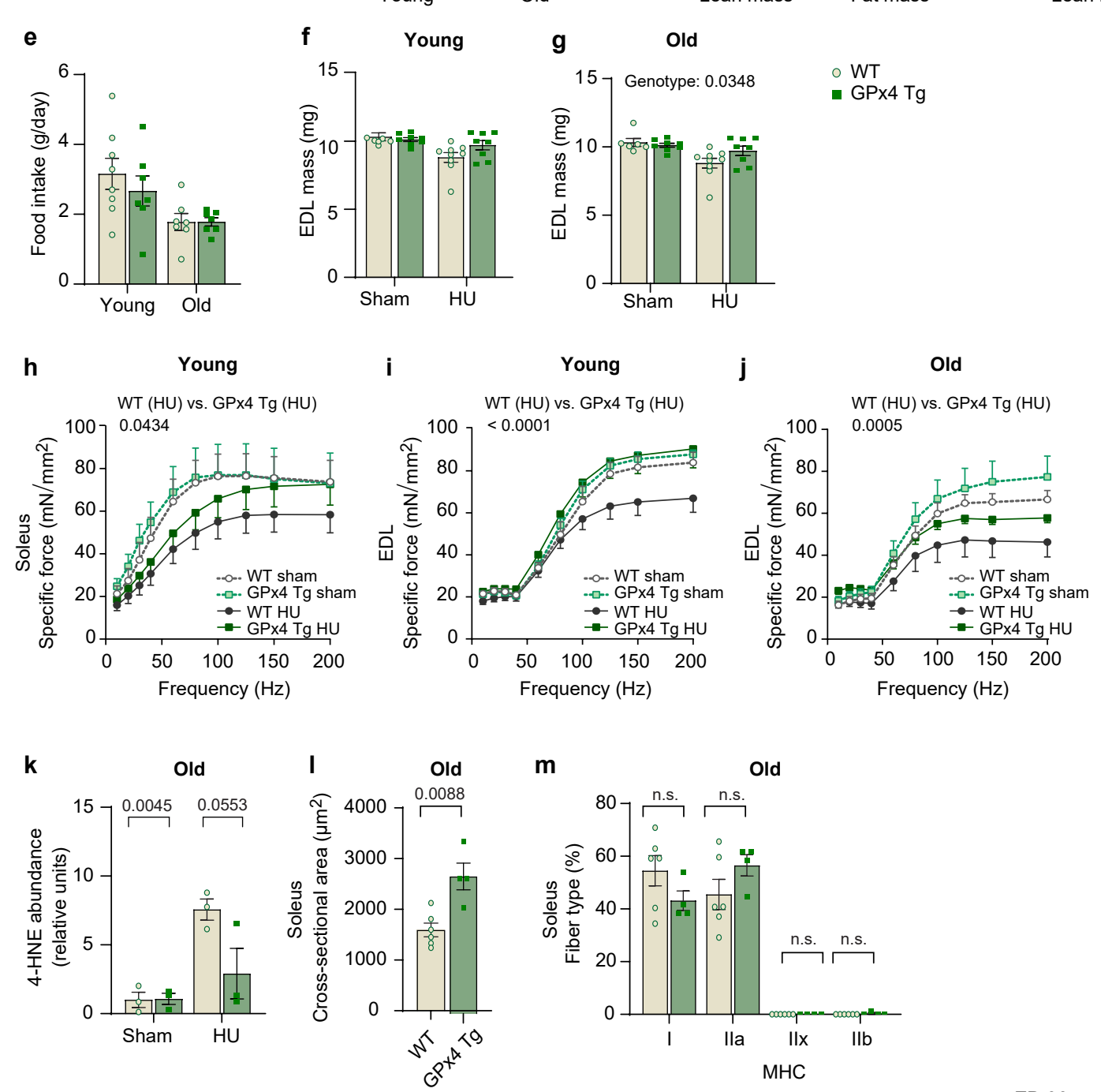

ED 06 


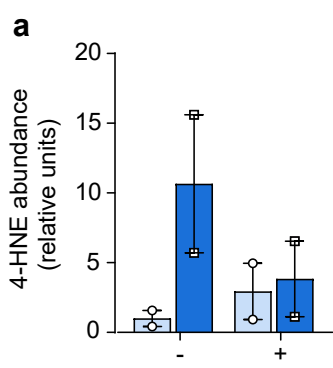

Ferrostatin-1

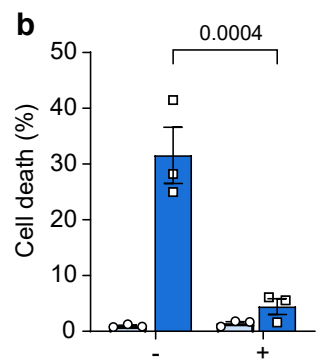

Ferrostatin-1

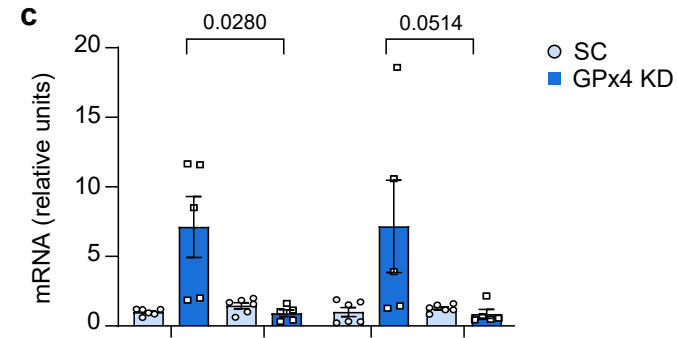

Ferrostatin-1 $\frac{-}{\text { CHAC1 }} \frac{-}{\text { PTGS2 }}$ d

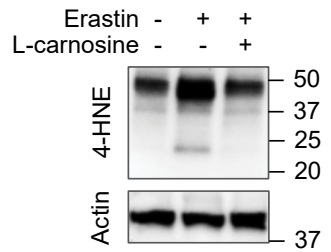

(kDa) e

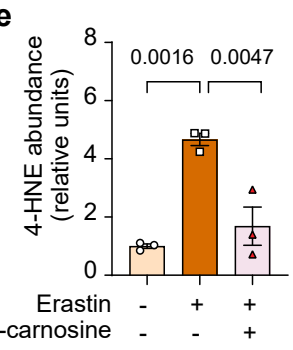

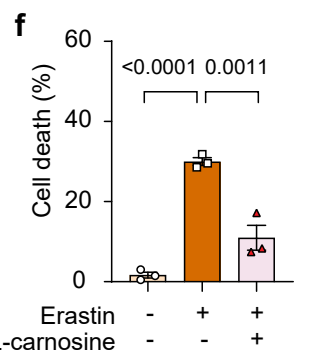

g GPx4 KD - $\quad-\quad+\quad+$
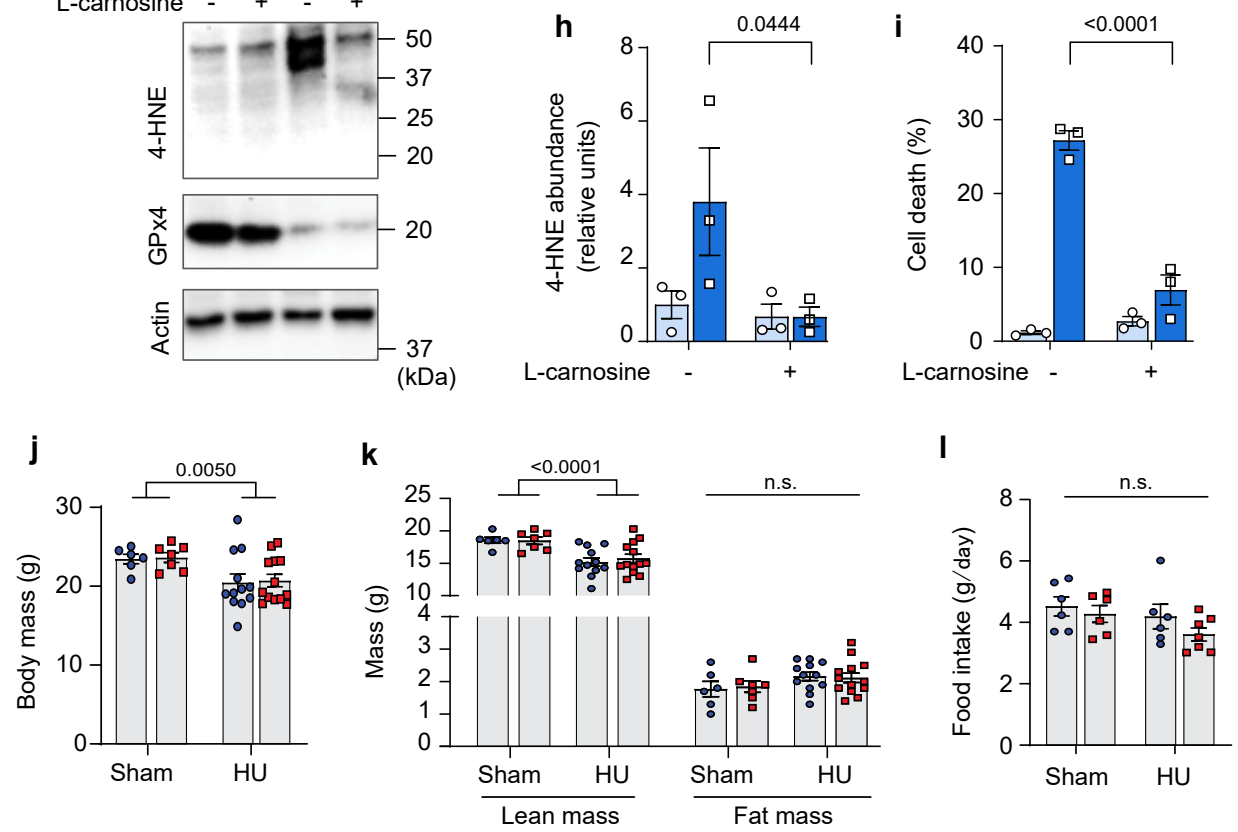

○ SC

- GPx4 KD

L-carnosine -
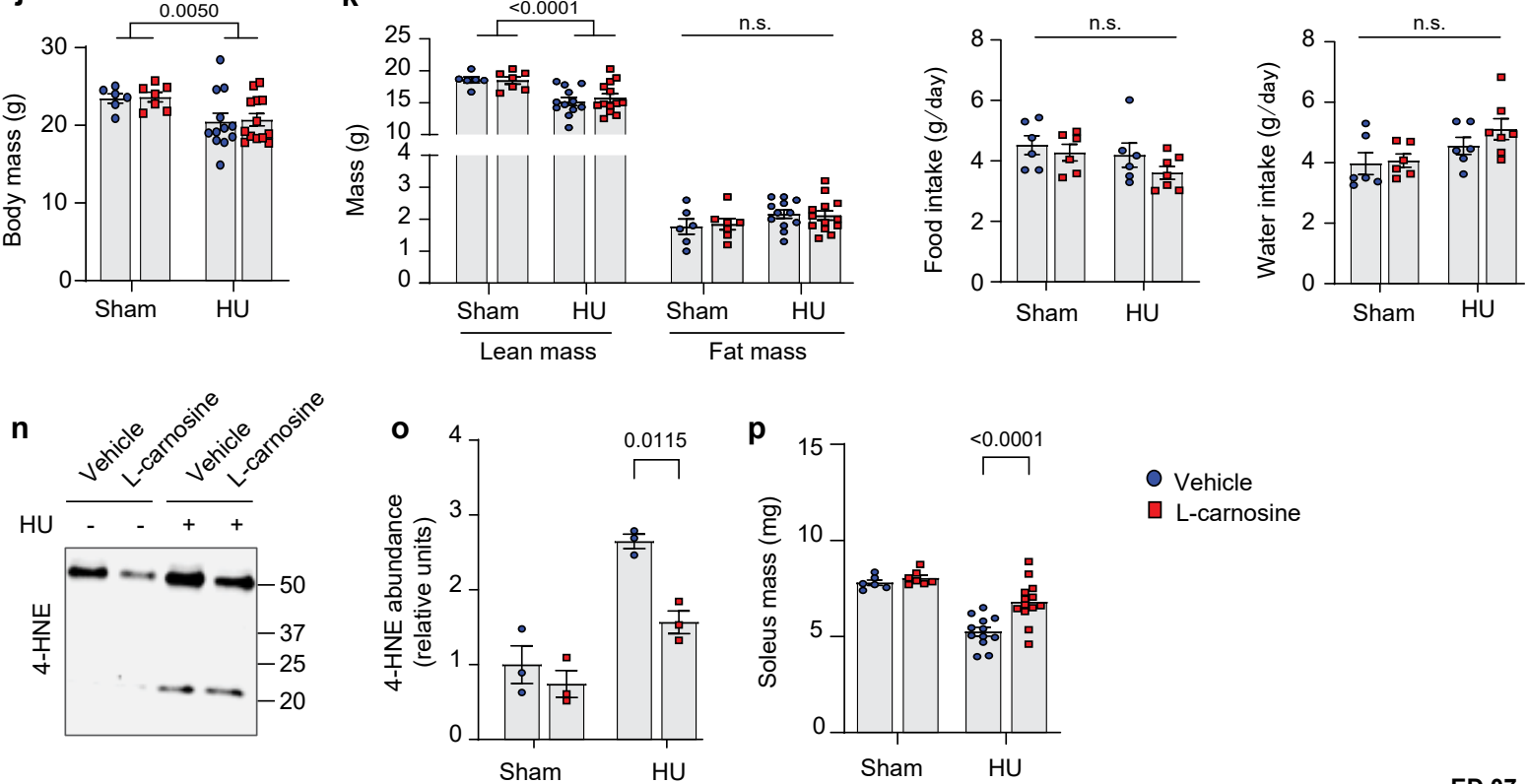

O Vehicle

$\square$ L-carnosine

ED 07 
a

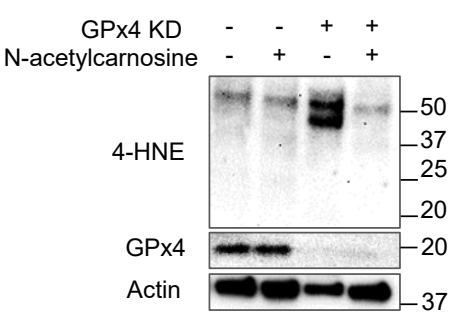

(kDa)

d

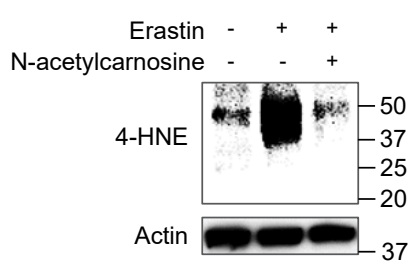

(kDa) b
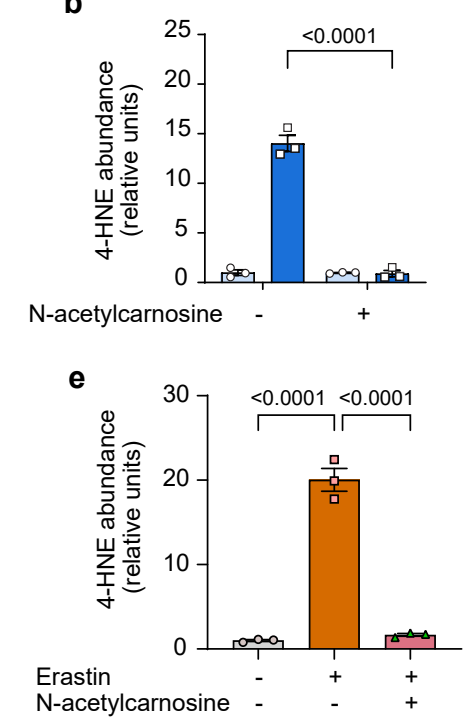

c

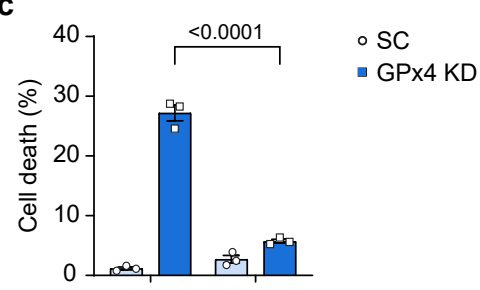

$\mathrm{N}$-acetylcarnosine - +

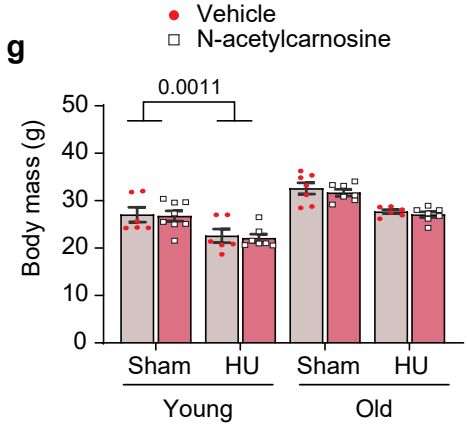

h

Young

i
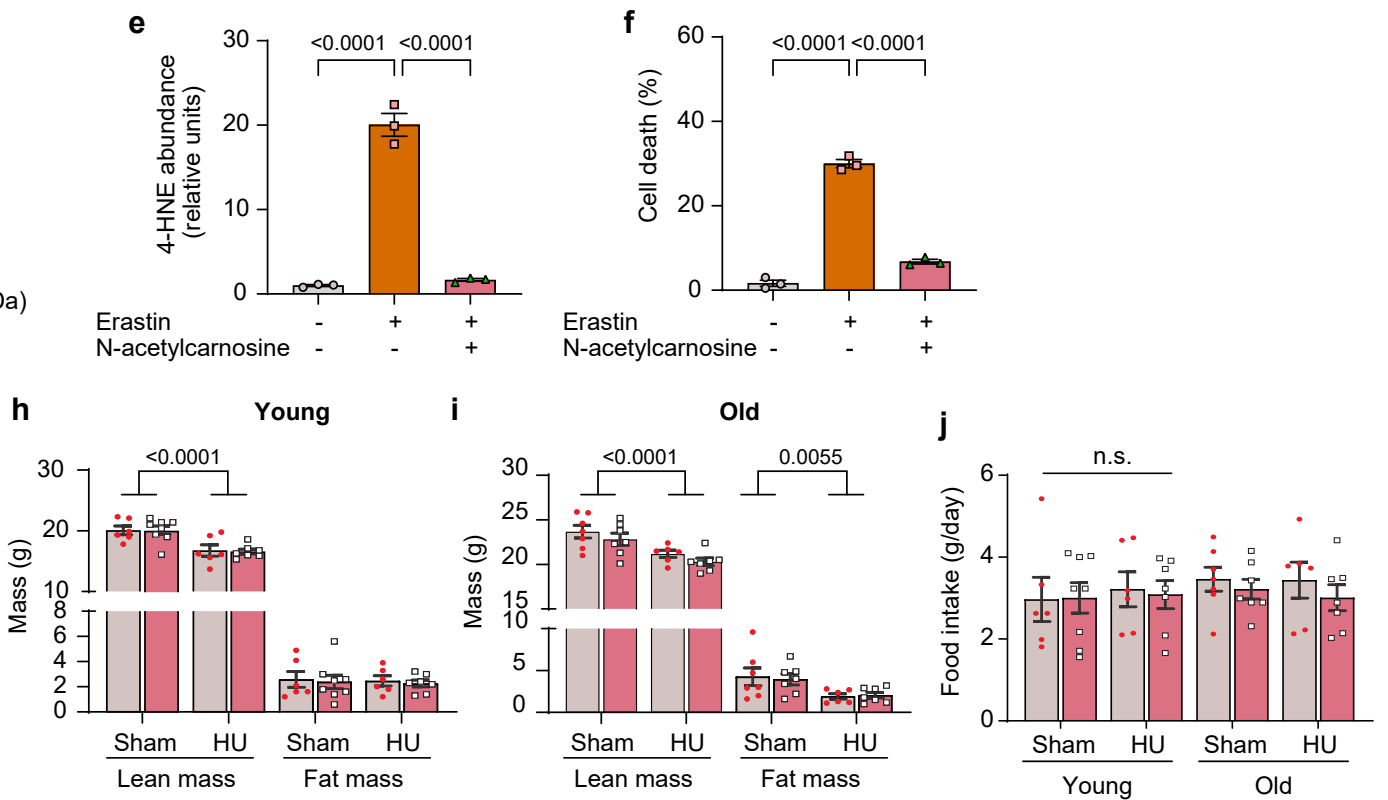

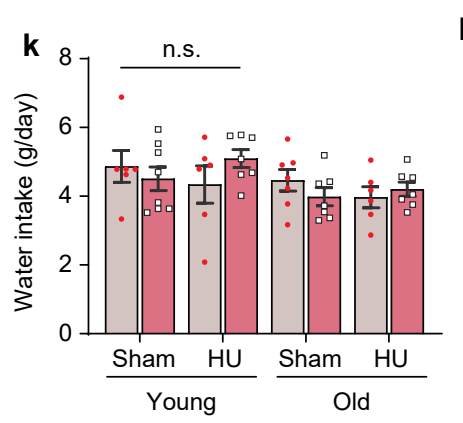

I

\section{Young}
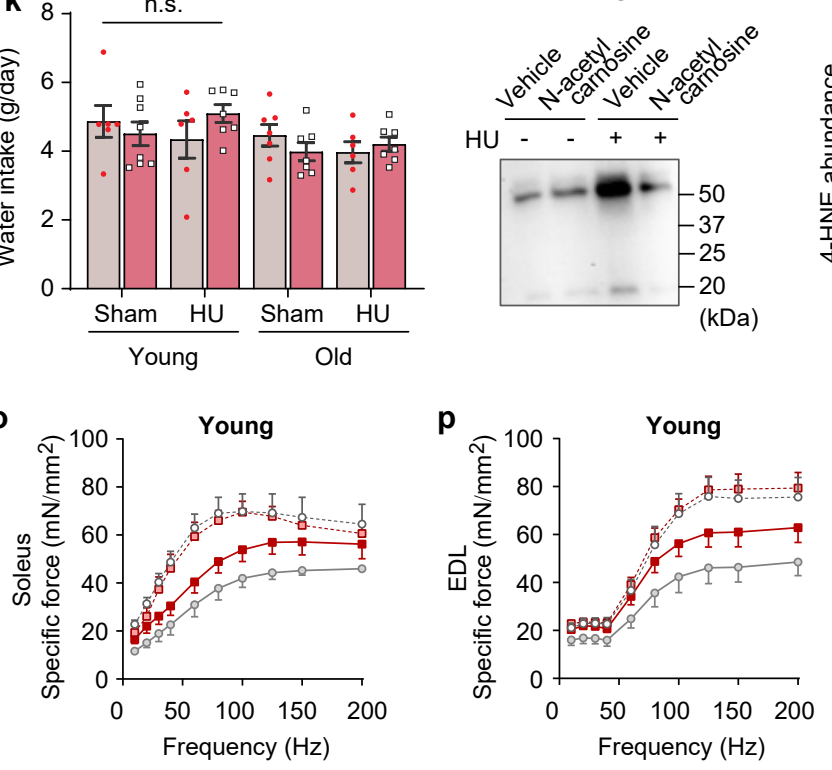

m
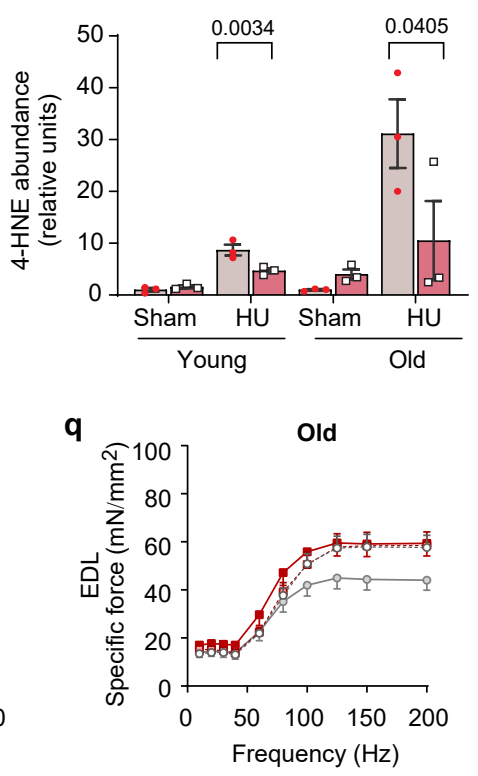

n

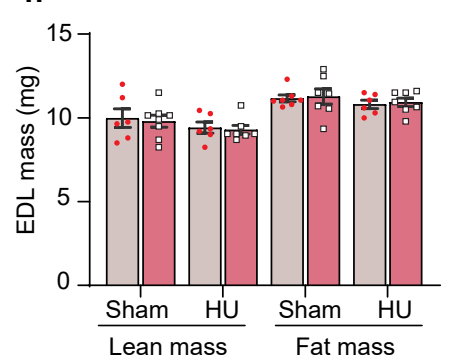

-o. Vehicle sham

- -- N-acetylcarnosine sham

-o- Vehicle HU

$\rightarrow \mathrm{N}$-acetylcarnosine $\mathrm{HU}$

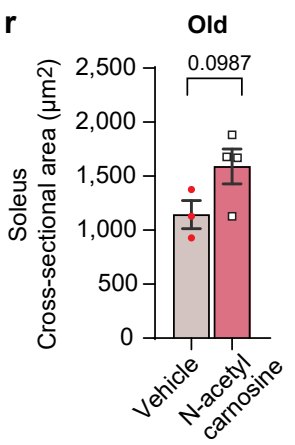

s

Old

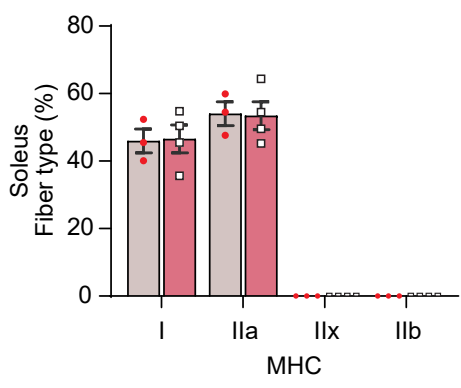

ED 08 
a

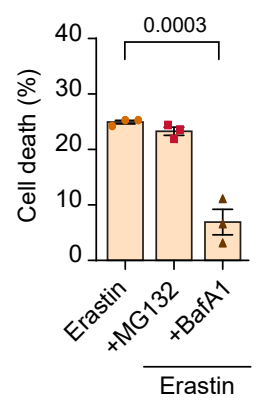

$$
\text { ○ } \mathrm{SC}
$$
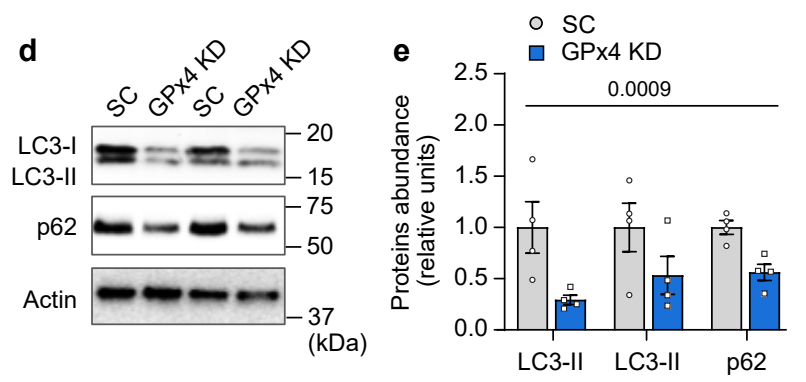

i

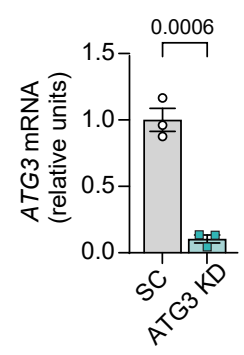

j

$\circ \mathrm{SC}$

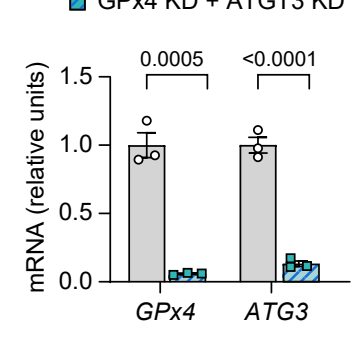

b

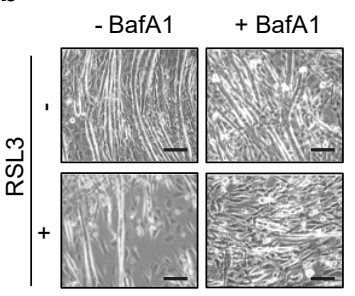

c

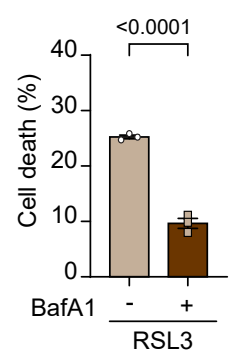

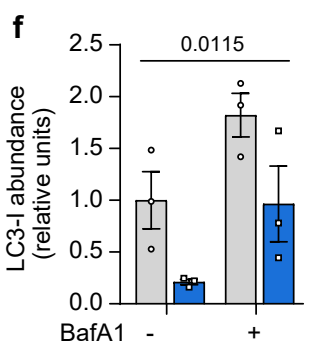
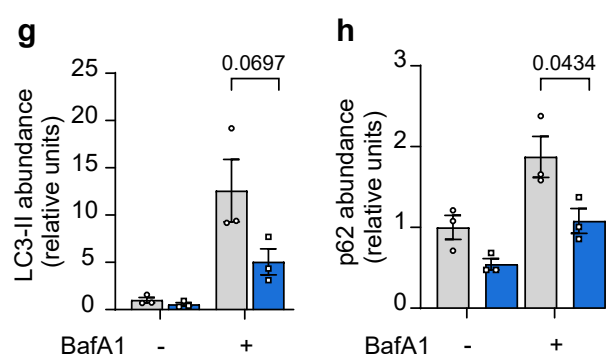

I $\circ \mathrm{SC}$

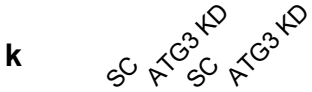

LC3-II 20

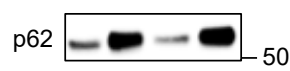

GPx4 -20

Actin $=-37$

(kDa)

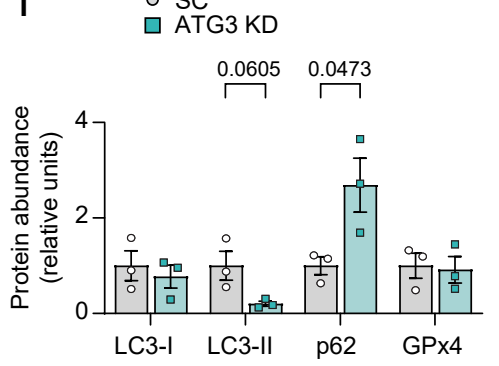



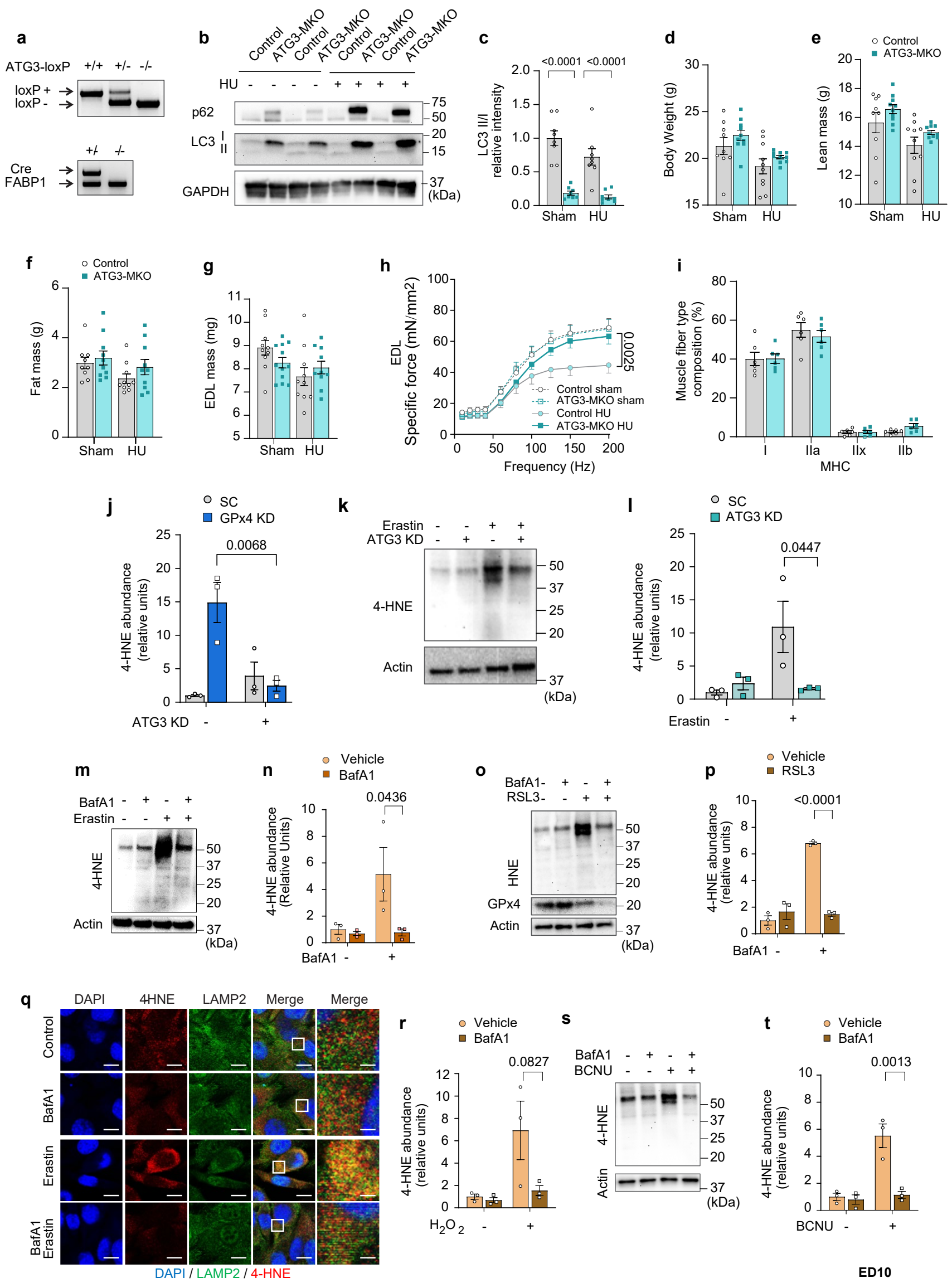

K. Funai, et al. 